\title{
Modeling and Simulation of Opinion Natural Reversal Dynamics with Opinion Leader Based on HK Bounded Confidence Model
}

\author{
Renbin Xiao $\mathbb{D}^{1,2}$ Tongyang Yu ${ }^{10},{ }^{3}$ and Jundong Hou ${ }^{4}{ }^{4}$ \\ ${ }^{1}$ School of Artificial Intelligence and Automation, Huazhong University of Science and Technology, Wuhan 430074, China \\ ${ }^{2}$ Key Laboratory of Image Processing and Intelligent Control (Huazhong University of Science and Technology), \\ Ministry of Education, Wuhan 430074, China \\ ${ }^{3}$ School of Management, South-Central University for Nationalities, Wuhan 430074, China \\ ${ }^{4}$ School of Economics and Management, China University of Geosciences, Wuhan 430074, China \\ Correspondence should be addressed to Renbin Xiao; rbxiao@hust.edu.cn and Jundong Hou; houjundong@cug.edu.cn
}

Received 30 September 2019; Revised 12 January 2020; Accepted 29 February 2020; Published 28 March 2020

Academic Editor: Marcelo Messias

Copyright (c) 2020 Renbin Xiao et al. This is an open access article distributed under the Creative Commons Attribution License, which permits unrestricted use, distribution, and reproduction in any medium, provided the original work is properly cited.

\begin{abstract}
Opinion natural reversals are important and common phenomena in network management. It is a naturally emerging process of opinions characterized by interactions between individuals and the evolution of attitudes themselves. To explore the underlying mechanism of this social phenomenon and to reveal its dynamic traits, we propose here a novel model which takes the effects of natural reversal parameter and opinion interaction on the individual's view choice behavior into account based on the Hegselmann and Krause (HK) bounded confidence model. Experimental results show that the evolution of individual opinions is not only influenced by the interactions between neighboring individuals but also updated naturally due to individual factors themselves in the absence of interaction, which in turn proves that the proposed model can provide a reasonable description of the entire process of public opinion natural reversal under the Internet environment. Besides, the proportion of group opinion tendency, network topology, identification method, and the influence weight of opinion leader will play significant roles in this process, which further indicates our improved model is very robust and thus can provide some insightful evidence to understand the phenomena of opinion natural reversal.
\end{abstract}

\section{Introduction}

The rapid advance of Internet technology and Web 2.0 has given rise to the wide presence of public opinion through the digital media. Unlike the one-way transmission context of traditional media, this type of new platform endows common individuals to share their opinions punctually and conveniently and thus makes social public opinion represent the rapid propagation speed and a wider influence scope. It has resulted in a lot of difficulties for social and cyber governance. As a result, the study of online public opinion has recently received much more attention in many fields. It can be found that a large number of online public opinions are often induced by social emergencies, in which some opinions are in the mainstream for a long time during the diffusion process, while there are also some opinions which are most likely to reverse suddenly for the slight change in certain individuals' thoughts. This emergent social phenomenon is called opinion reversal [1], and the documentary Under the Dome released by the Chinese journalist Chai Jing in 2015 is one of the most famous examples.

To the best of our knowledge, some opinion reversal phenomena are caused by human control, and others have resulted from the diffusion mechanism of the opinions themselves. The latter is a so-called natural reversal of opinion in this paper and is also referred to as public opinion reversal in some cases. In nature, the choice of individual opinion is a decision-making behavior which is affected by 
not only external situations but also internal cognition related to the decision itself [2]. Accordingly, opinion natural reversal is an evolutionary process of viewpoint natural mutation caused by the shift of individual cognition which is resulted from both the information interaction with other participators and the psychology of the involved persons themselves. It is not the result of forced reversal by external intervention. It indicates that the selection and evolution of individual opinions are the internal determinants of natural reversal and the driving forces of public opinion orientation. Therefore, it has been observed that understanding this kind of opinion reversal is beneficial for monitoring and early warning of online public opinions and exploring the dynamics of opinion natural reversal will contribute to improving the network emergency response and the social governance capability of the corresponding governmental departments. However, in order to unfold the underlying mechanism of this complicated social phenomenon, it is necessary to propose a suitable model to understand the inherent dynamic characteristics in the evolution process of opinion natural reversal.

The states of individual and collective opinions always change dynamically with the information acquired from the whole society and their surrounding environment, which leads to different behavior patterns of groups from the macroperspective. Social physicists have employed the models and methods in information science and complex network to understand this type of evolution process. It is well-known that opinion dynamics models originated in the study of social dynamics that aims to explore the evolution rules of group states over time in the social system. Similarly, opinion natural reversal is most likely to reverse suddenly due to the slight change in certain individuals' ideas. Substantially, this emergent social phenomenon could be examined and simulated by using the opinion dynamics model. However, among prior studies about opinion dynamics, most researchers focused on exploring the macrolevel emergent behavior from the perspective of microlevel mechanism, such as the opinion dynamics model in the field of statistical physics [3]. These models were mainly to reveal the opinion evolution based on the interaction roles between particles in physics [4]. Although prior models have provided the needed quantitative ways for conducting studies involving the formation and propagation mechanism of public opinion, they were based on the premise that the evolution law of ideas is subject to the linear interaction, and the processing methods were limited to the traditional methods including matrix, Markov chain, and graph theory. Furthermore, a pair of individuals actually begin to affect each other when the difference between their opinions is below a given threshold, and the existing models failed to take this nonlinear interaction into account. Subsequently, with the development of complexity science, the nonlinear opinion dynamics models which are more in line with the actual situation were gradually proposed with the tools of social networks [5] and social dynamics. Since the analytical solution of this kind of model is often difficult to get in most cases, computer simulation has become the main method to resolve this problem $[3,6,7]$. Currently, the dynamic models can be roughly divided into two categories: the master equation model of stochastic social systems [8] and the multiagent systems model of deterministic social systems [9]. The former can only be solved by a numerical method, while the latter is generally adopted if the size of the network scale is a finite natural number $N$; the dynamic change in individual's state needs to be considered from a microlevel.

Another major line of previous works focused on whether opinions are discrete or not. Discreteness means individual opinion is captured by a discrete integer value; for example, $0,+1$, and -1 can be used to denote the neutral, positive, and negative opinions, respectively. The typical models include the Ising Model [10, 11], Sznajd Model [12], and majority rule model [13]. It has been found that the discrete model cannot describe the continuous transitions in public opinions; thus, the idea of continuous value (i.e., a number between 0 and 1) capturing an individual opinion is applied in modeling of public opinion. Among them, Deffuant and Weisbuch proposed a multiagent system (DW model) to check the interdependence between the similarity of agents' opinions and the frequency of their contacts $[14,15]$, and Krause and Hegselmann advanced a relative agreement model (HK model) quite similar to the former one $[16,17]$. The bounded confidence rules indicate that a pair of agents begin to impact each other when the difference between their opinions is not higher than a given threshold [18], and the evolution of collective opinions may eventually lead to three stable states: consensus, polarization, or separation [19]. Both the DW model and the HK model are repeatedly updated with the average value of opinion based on bounded confidence at discrete time points, and this rule conforms to the "selective contact" in social psychology [20]. However, their differences lie in the range and mode of individual communication, and at a specific time, only a pair of agents which meet the confidence threshold will randomly interact in the DW model, while all individuals who satisfied this condition can communicate their views at the same time in the HK model, and the state of each agent's opinion is always updated to the average value of all individuals within their confidence threshold. Recently, these two models have received great attention in the field of opinion dynamics and have improved and extended for the specific phenomena and problems in order to reasonably evolve the public opinion. However, the improvements based on the DW model mainly included reciprocity feedback consistency [21], first impression effect under general opinion distributions [22], interaction selection rules [23], steady-state property in social networks [24], social learning with heterogeneous agents [25], dynamics of bounded confidence threshold [26], noise impact [27], and interpersonal network [28]. The expansions of the $\mathrm{HK}$ model were reflected in confidence threshold [29], nonlinear viewpoint updating rules [30], opinion leaders [31], trust threshold heterogeneity [32], directed network [33], self-confidence parameter [34], and cognitive styles [35].

Although the classical opinion dynamics model of bounded confidence mentioned above has simplified the interaction rules and the selection of interaction objects for the purpose of studying the emerging macroscopic behaviors 
of groups and has achieved promising results in modeling of public opinion, collective opinion reversal is often the result caused by the combination roles of internal factors (i.e., individual psychological and cognitive heterogeneity) and external influences (i.e., opinion leaders [36] and social interaction network structure [37]). Therefore, there are some gaps that need to be solved yet: (1) as we all know, the communications between individual agents are, in general, very beneficial to achieve consensus in public opinion formation in the real-life, and this demonstrates that both the bounded confidence hypothesis and its repeated average updating rule have real-social context, which in turn means the dynamics model based on bounded confidence is suitable for exploring the natural reversal of online public opinion. However, the traditional HK model concentrated on the phenomenon of consistency and differentiation in the steady state of opinion diffusion under simple rules, as well taken the influence of individual interaction on opinions into consideration, but there still remains little work that systematically considers and distinguishes whether a node is influenced by opinion leaders or ordinary individuals. Generally, opinion leader is obviously the critical node with a higher centrality in social networks, and it is bound to affect public opinion assimilation, integration, and separation, but his/her influence on opinion evolution is obviously different from that of ordinary individual, so the classical HK model cannot explain this problem. (2) Even though some studies considered the role of opinion leader in the opinion evolution, they tended to randomly select the node as an opinion leader node from the social network. But in fact, opinion leaders should be the established nodes in the real scenario, which requires to accurately identify them. Otherwise, it is difficult to guarantee the authenticity of opinion evolution. (3) The traditional opinion dynamic models held that the viewpoints in the whole system were neither attenuated nor augmented, which is insufficient in description of opinion natural reversal. The reason is although the original HK and its extended models considered the impact of individual interaction on opinion diffusion, they did not take into account that the participator's view itself also may be changing dynamically. Actually, individuals will enrich their own knowledge and improve their experiences by means of web page browsing and information receiving, which will unconsciously impact their cognition of a specific event and opinion choice. This is an objective phenomenon that individual's opinion itself usually shifts with time even without the interaction effect of neighbor nodes, while little is known about which parameter can perfectly describe this trend of opinion evolution over time. To fill in these gaps, our study employs the centrality index in SNA to identify the opinion leaders and proposes a modified dynamics model for the opinion natural reversal based on HK bounded confidence model considering the natural reversal features caused by individual cognition in the network environment. It is used to put particular emphasis on understanding the phenomenon of opinion natural reversal and its internal mechanism in the evolutionary process of public opinion.

The rest of this paper is organized as follows. The centrality index in SNA is proposed to measure the opinion leaders in the group, and a leader-follower opinion dynamic model with natural reverse and heterogeneity is advanced in Section 2. In Section 3, we report some computer simulation results about the evolution of public opinion and discuss the phenomenon about opinion natural reverse. Section 4 is further to check the sensitivity and robustness of our model. We conclude this study and give some potential research directions in Section 5.

\section{Opinion Natural Reversal Dynamics Model}

The HK model gives the influence of the agents' behaviors on the evolution process, and the agents in this model have the same behaviors; that is, they all update their own opinions according to the bounded trust rules, without considering other situations in opinion dynamics, such as the influence of opinion leaders $[38,39]$.

2.1. HK Model. To construct the HK model, we consider $n$ agents in a system, the set of which is denoted as $A=\{1,2$, $\ldots, n\}$. The opinion of agent $i$ at time $t$ is represented by $x_{i}(t) \in[0,1]$, and a vector is formed $x(t)=\left(x_{1}(t), x_{2}(t)\right.$, $\left.\ldots x_{n}(t)\right), i \in A, t \geq 0$. Each agent has an initial opinion $x(0)$ at $t=0$. According to the HK model,

$$
I(i, x(t))=\left\{1 \leq j \leq n|| x_{i}(t)-x_{j}(t) \mid<\varepsilon_{i}\right\},
$$

where $I(i, x(t))$ is a set of all individuals within the confidence threshold of agent $i$ at time $t, \varepsilon_{i}$ is the agent $i$ 's threshold of confidence, and $\left|x_{i}(t)-x_{j}(t)\right|<\varepsilon_{i}$ denotes the difference between the opinion of agent $i$ and the opinion of its neighbor $j$. When the difference is less than the confidence threshold, the agent will choose to interact with its neighbors.

To simplify the problem, these agents within the trust boundary are given the same weight, and the weight of agents outside the boundary is valued 0 . Formula (2) gives the specific form of the HK model:

$$
x_{i}(t+1)=|I(i, x(t))|^{-1} \sum_{j \in I(i, x(t))} x_{j}(t), \quad t \in T,
$$

where $|\cdot|$ represents the number of agents within the boundary. HK model is studied for the evolution of ideas over a period of time within discrete time, $T=\{0,1,2, \ldots\}$. The final state of the dynamical system changes with different $\varepsilon_{i}$.

2.2. Opinion Leaders and Centrality. Opinion leaders have a great influence on the diffusion of opinions, which will affect the whole dynamic process of opinions. Opinion leaders refer to "activists" who have high influence and appeal in the social network of the system and often provide information or opinions to others and exert influence on them. Opinion leaders can influence the formation of group opinion and value judgment, as well as the diffusion trend of online public opinion. Therefore, this paper introduces the influence of opinion leaders into the opinion dynamics model as 
a starting point to study the internal mechanism of public opinion structure reversal.

The traditional practice is to randomly assign some individuals as opinion leaders in the simulation system [40]. As we know, opinion is spread in the social system, its social relation network structure characteristics also affect diffusion [41], and the opinion leader is the key node with more connections in social networks; thus, it is obviously difficult to accurately reflect the objective reality in the social system by randomly setting opinion leader nodes. In order to better identify the opinion leaders in the group, this paper attempts to adopt the social network analysis method. The specific reason is that social network represents the collection of social actors and their relations and emphasizes the relationship between each actor and others. It is a method to analyze the structure and properties of social relations and to determine the crucial nodes from the social system based on the dynamic characteristics of agents and the network background. In general, the key node's measures of social network analysis contain the three indexes of degree centrality, closeness centrality, and betweenness centrality [42].

Degree centrality is measured by the total amount of direct links with the other nodes. Its formula is shown as follows:

$$
C_{i}=\frac{m}{(n-1)}
$$

where $m$ is the number of nodes connected with agent $i$ and $n$ is the number of agents in a network. In the subsequent analysis, centrality is short for degree centrality in our study without being specially explained.

Closeness centrality is to describe the ability of an agent who is not controlled by other agents, and it can be calculated by the following equation:

$$
C_{i}=\frac{n-1}{\sum_{j \neq i} d_{i j}},
$$

where $d_{i j}$ is the average distance from node $i$ to $j$.

Betweenness centrality is to measure one node undertaking "mediation" role in a network and can be computed by the following equation:

$$
C_{i}=\sum_{i \neq j \neq k} \frac{g_{j k}(i)}{g_{j k}},
$$

where $g_{j k}(t)$ is the number of shortest paths going through node $i$ from node $j$ to $k$ and $g_{j k}$ means the amount of all shortest paths from node $j$ to $k$ in a network.

2.3. Opinion Natural Reversal Model. Combining opinion leaders into the HK model to explore the dynamic mechanism regarding the natural reversal of opinions, the following model is proposed.

$x_{i}$ is the agent $i$ s opinion, which valued between $[-1,1]$ to quantify the opinion on the event. $[0.2,1]$ generally represents the recognition or positive attitude towards the event, $[-1,-0.2]$ represents the opposite or negative opinion towards the event, and $(-0.2,0.2)$ represents the relatively neutral opinion.

The effect of opinion leader on opinion diffusion dynamics is obviously different from that of the ordinary group since an opinion leader can influence ordinary groups by its status in social network, and opinion leader's viewpoint is usually not neutral. Accordingly, when proposing the model, individual agents participating in the evolution of views are divided into two categories: ordinary group and opinion leaders. Opinion leaders can be identified with the centrality index of SNA as mentioned above, and the rest is regarded as ordinary agents. With the evolution of time, each agent interacts with other connected agents, and its interaction rules are carried out according to the HK model. However, whether the neighbor agent is an opinion leader or an ordinary agent, the influences produced are different. Therefore, considering the heterogeneity between opinion leaders and ordinary agents [43], corresponding diffusion dynamics models are designed as follows, respectively.

First of all, a $n \times n$ matrix $R$ is to represent the relationship matrix between individuals in a social network. For all individuals $i$ and $j$ in $A, R_{i j}$ means whether individual $i$ is connected with $j$. When $R_{i j}=0$, it denotes individual $i$ is not connected with $j$; and when $R_{i j}=1$, there is a link between them. Then, supposing the amount of opinion leaders in a network is $m, m \in B=\{1,2, \ldots, m\}$. Thus, the number of ordinary individuals is $n-m$, belonging to set $C=\{m+1$, $m+2, \ldots, n\}$. Certainly, $A=B \cup C$. The rules of opinion updating are as follows.

\subsubsection{Opinion Evolution Modeling of Ordinary Group. If} agent $i$ is an ordinary individual, then $i \in C$. Traditionally, agent $i$ is impacted by its neighbor nodes, while its neighbor may be either an opinion leader or an ordinary individual. In order to take multiple effects into consideration comprehensively, ordinary group opinion dynamic evolution is modeling by the linear weighted rules of the joint influences for these two groups as follows:

(1) Calculating the sets regarding the trust of ordinary individuals and opinion leaders within the confidence threshold of agent $i$ at time $t$ :

$$
\begin{aligned}
& L(i, x(t))=\left\{1 \leq j \leq m|| x_{i}(t)-x_{j}(t) \mid<\eta_{i}, j \in B\right\}, \\
& I(i, x(t))=\left\{m+1 \leq j \leq n|| x_{i}(t)-x_{j}(t) \mid<\varepsilon_{i}, j \in C\right\},
\end{aligned}
$$

where $\eta_{i}$ means the confidence threshold of agent $i$ to the opinion leaders and $\varepsilon_{i}$ represents the confidence threshold of agent $i$ to the ordinary individuals. $L(i$, $x(t))$ and $I(i, x(t))$ denote the sets of opinion leaders and ordinary individuals within the confidence threshold of agent $i$ at time $t$, respectively.

(2) According to the connection relationship of nodes in a social network, determining the specific agents $j$ 
linked with agent $i$ in the sets of $B$ and $C$, agent $i$ 's opinion at time $t+1$ will be influenced by the agent $j$ that is within the corresponding confidence threshold, marked $b_{i j}(t)$ and $c_{i j}(t)$, respectively:

$$
\begin{aligned}
& b_{i j}(t)= \begin{cases}1, & \text { if } R_{i j}=1, j \in L(i, x(t)), \\
0, & \text { otherwise, }\end{cases} \\
& c_{i j}(t)= \begin{cases}1, & \text { if } R_{i j}=1, j \in I(i, x(t)), \\
0, & \text { otherwise, }\end{cases}
\end{aligned}
$$

where the value of $b_{i j}(t)$ indicates whether the viewpoint of agent $i$ will be impacted by the opinion leader $j$ within the confidence threshold $\eta_{i}$. When $j \in L(i, x(t))$ and $R_{i j}=1$ and $b_{i j}(t)=1$, the opinion leader $j$ will influence the viewpoint of agent $i$. Otherwise, there is no effect. Similarly, the value of $c_{i j}(t)$ indicates whether the viewpoint of agent $i$ will be impacted by the ordinary individual $j$ within the confidence threshold $\varepsilon_{i}$. When $j \in I(i, x(t))$ and $R_{i j}=1$ and $c_{i j}(t)=1$, agent $i$ will be affected by the ordinary agent $j$. When $c_{i j}(t)=0$, agent $j$ has no effect on agent $i$.

(3) Based on trust set and matrix $R$, the opinion of agent $i$ will be updated as the following equation:

$$
\begin{aligned}
x_{i}(t+1)= & \theta \frac{1}{\sum_{j \in L(i, x(t))} b_{i j}(t)} \sum_{j \in L(i, x(t))} b_{i j}(t) x_{j}(t) \\
& +(1-\theta) \frac{1}{\sum_{j \in I(i, x(t))} c_{i j}(t)} \sum_{j \in I(i, x(t))} c_{i j}(t) x_{j}(t),
\end{aligned}
$$

where $\theta$ is to measure the influence power of opinion leaders, while the influence power of the ordinary group is $1-\theta$. If there is no opinion leader, let $\theta=0$, the model is the HK model, all the agents belong to the ordinary group, and their opinions evolve according to the HK model. When $\theta>0$, both the ordinary group and the opinion leader group are in the whole system. Individuals will update their opinions comprehensively considering the joint effects of ordinary agents and opinion leaders. Additionally, $\theta=1$, this is to illustrate individual $i$ is only affected by opinion leaders, and the role of ordinary individual is not considered.

(4) If the opinions of all individuals do not continue to change, or the following condition is met, the update will be ended:

$$
\sum_{i=1}^{n}\left[x_{i}(t+1)-x_{i}(t)\right]^{2} \leq \delta,
$$

where $\delta$ is set as a positive number close to 0 . In our study, we let it equal to 0.0001 .

2.3.2. Opinion Evolution Modeling of Opinion Leader Group. If agent $i$ is an opinion leader, then $i \in B$. Since opinion leader agents are more likely to insist on their own views, they may only be influenced by other opinion leaders with a certain authority, rather than the ordinary agents in the short run. Hence, the diffusion dynamics process could be varied with the initial attitudes of opinion leaders. In a general way, there are two categories of initial attitudes of opinion leaders, positive and negative. Accordingly, the evolution of viewpoints of opinion leaders with a positive attitude can be described by formula (10), and the opinion update law of opinion leaders with a negative attitude is given by formula (11):

$$
\begin{gathered}
x_{i}(t+1)=\theta|L(i, x(t))|^{-1} \sum_{j \in L(i, x(t))} x_{j}(t)+(1-\theta) \times 1, \\
x_{i}(t+1)=\theta|L(i, x(t))|^{-1} \sum_{j \in L(i, x(t))} x_{j}(t)+(1-\theta) \times(-1),
\end{gathered}
$$

where $x_{j}(t)$ denotes the viewpoint of an opinion leader.

2.4. Natural Reversal Parameter. To describe the process in which individual opinion itself will naturally vary across time, even if there is no interactive influence of individual neighbors, it is necessary to introduce a parameter that changes with evolution time to modify the HK model. The theories regarding individual complexity and personality trait emphasized that the individual's psychological cognition ability will enhance with the increase in knowledge and experiences. This indicates that the natural reversal parameter is not a fixed constant but a monotonically increasing function. In the early stage of evolution, due to the limited knowledge of an individual, the value of this parameter should be small. However, with the increase in time, the knowledge and experiences are more abundant, and the individual's opinion choice may not be steadfast, so the value of this parameter will become larger. The property of this parameter is similar to the Sigmoid function:

$$
s(x)=\frac{1}{\left(1+e^{-x}\right)},
$$

where the value of $x$ can be any real number, $s(x) \in(0,1)$, which can map a real number to the interval of $(0,1)$. This function will increase monotonously within the real number range $x \longrightarrow-\infty, s(x) \longrightarrow 0$, and $x \longrightarrow+\infty, s(x) \longrightarrow 1$.

However, when a natural reversal parameter is designed according to the properties of Sigmoid function, the microscopic instantaneous transformation cannot be reflected. Therefore, the derivative is calculated:

$$
s^{\prime}(t)=\frac{e^{-t}}{\left(1+e^{-t}\right)^{2}} .
$$


Based on equation (13), considering the basic process and critical influencing factors of natural reversal of opinion, the natural reversal parameter $\alpha(t)$ is specially constructed as shown in the following equation:

$$
\alpha(t)=\frac{\mu e^{1 / \beta(t-\gamma)}}{\left(1+e^{1 / \beta(t-\gamma)}\right)^{2}}
$$

where $\alpha(t)$ increases with time $t$ and $0<\alpha \leq 1 . \mu$ is used to illustrate the impact index of $\alpha(t)$, and it is usually equal to 4 . $\beta$ denotes the evolution rate, and $\beta \in[1,4] . \gamma$ represents the opinion decay time and is ranged from 10 to 30 .

Therefore, after introducing parameter $\alpha$, the opinion update modeling for the ordinary agent $i$ is as follows:

$$
\begin{aligned}
x_{i}(t+1)= & \alpha(t)\left(\theta \frac{1}{\sum_{j \in L(i, x(t))} b_{i j}(t)} \sum_{j \in L(i, x(t))} b_{i j}(t) x_{j}(t)\right. \\
& \left.+(1-\theta) \frac{1}{\sum_{j \in I(i, x(t))} c_{i j}(t)} \sum_{j \in I(i, x(t))} c_{i j}(t) x_{j}(t)\right) .
\end{aligned}
$$

Correspondingly, the opinion update equations for the opinion leaders with positive and negative attitudes are formulas (16) and (17), respectively:

$$
x_{i}(t+1)=\alpha(t)\left(\theta|L(i, x(t))|^{-1} \sum_{j \in L(i, x(t))} x_{j}(t)+(1-\theta) \times 1\right) \text {, }
$$

$$
x_{i}(t+1)=\alpha(t)\left(\theta|L(i, x(t))|^{-1} \sum_{j \in L(i, x(t))} x_{j}(t)+(1-\theta) \times(-1)\right) \text {, }
$$

in which the different values of parameter reflect the difference regarding the influence of this parameter on supporting or opposite groups whether they are ordinary groups or opinion leaders. The influence of specific parameters on evolution needs to be compared and tested in the simulation section.

\section{Numerical Simulation Results}

3.1. Simulation Scenario and Experimental Design. Traditionally, the evolution models regarding opinion diffusion and public opinion are mostly based on regular or random networks. However, compared with the total number of nodes or connections, in the network formed by individual participants, the shortest paths of any two nodes are very small, and its scale distribution of connected subgraphs is a typical power law distribution. Besides, since there are complex social network structures in the online incidents, all of them would be shifted from the simple random network to the complex nonrandom network [44]; therefore, the network formed by the agents in online public opinion should have some structural features of online social networks. Accordingly, in this paper, the default network is scale-free in the simulations to reflect an actual issue that public opinion is mainly based on the Internet or mobile network.

In this situation, our study simulated and analyzed the process of public opinion reversal using the proposed method. Essentially, as discussed above, "public opinion reversal" involves minor agents persuading the majority to change opinions. The size of agents (or nodes) is $n=1000$ in the social system simulated here according to the literature [1]. Each individual agent $i$ has a state value of initial opinion, and let $x_{i} \in[-1,1]$. In the initial case, the agents can be divided into three categories according to the opinion value: supporters $[0.2,1]$, opponents $[-1,-0.2]$, and neutrals $(0.2,0.2)$. Five nodes are initially set to represent the individuals who cause the discussion heat in the public opinion, and then we assume that there are two groups including opinion leader and ordinary participant. If the proportion of opinion leader is 0 , the proposed model is a $\mathrm{HK}$ model. Over time, the agents will interact with its neighbor nodes in the local network according to the principle of bounded confidence, and interactions should be carried out in accordance with the dynamic model of the natural reverse built in Section 2; opinion leaders will affect view evolution, and the system groups' view evolution will experience growth and natural reverse process to a steady state. The simulation runs until the opinion reaches a steady state or until at most 100 steps have passed. All simulation results were averaged over 100 runs.

In addition, it needs to be noted that the steady state of the system means that if the opinion of all individuals does not continue to change, the system becomes steady [45]. Moreover, the impact of these factors on the evolution of public opinion was measured by steady state and steady time of the social system. The process framework of simulation experiment design is as follows (Figure 1):

Step 1 (initialization): determining the evolutionary period and initializing parameters such as the number of nodes, confidence interval, convergence condition, and the number of simulation steps.

Step 2 (formation of social network): generating a needed network.

Step 3 (setting of initial opinion value): each agent is given an opinion $x_{i}(0)$, and the initial opinions of the agents in the system are subject to a particular distribution, such as a uniform or normal distribution.

Step 4 (identification of opinion leader): applying the social network analysis method to identify opinion leaders in the generated network. If Period $=1$, an opinion leader can be regarded as an agent with a positive attitude. If Period $=2$, this opinion leader can be set as an agent with a negative attitude.

Step 5 (calculation of natural reversal parameters): computing the natural reversal parameter of each node.

Step 6 (update of viewpoint): updating the value of opinion according to the dynamics model of different types of network nodes (opinion leaders and ordinary individuals). 


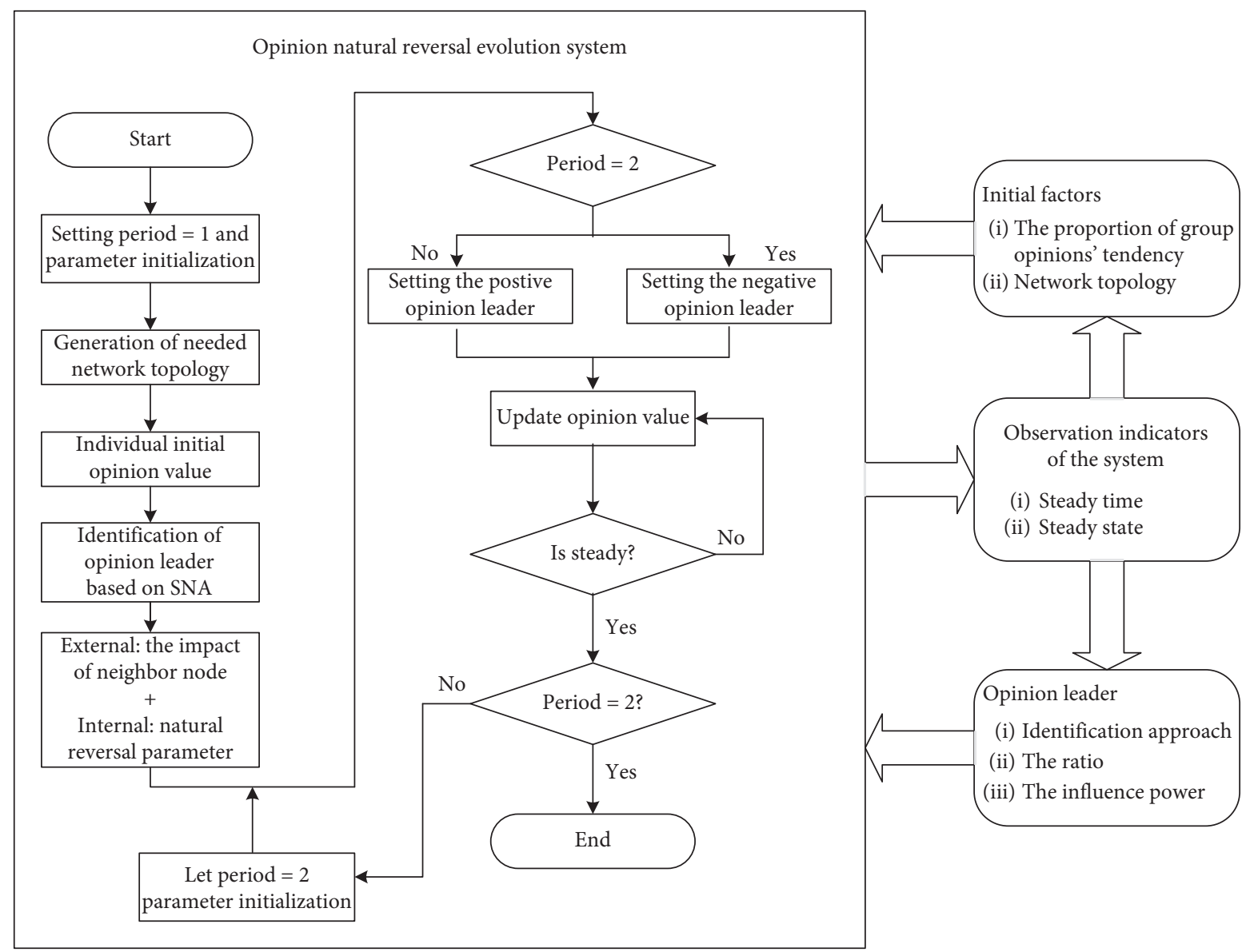

FIGURE 1: Algorithm process of the simulation experiment design.

Step 7 (judgment of the stable state of the system): if the system becomes steady, the simulation will be ended; otherwise, go back to step 4 .

Step 8 (judging whether Period is equal to 2): if Period is not equal to 2, let Period $=2$ and go back to step 4 . Otherwise, the simulation experiment is over.

Step 9 (verification for the impact of relevant parameters): experimentally testing the impacts of related parameters on the natural reversal process, including the proportion of group opinions' tendency, network topology, and the identification approach and influence power of opinion leader.

3.2. Identification of Opinion Leader. To identify the opinion leaders in the social system from 1000 nodes of a scale-free network, three indicators regarding centrality in social network analysis were applied according to Section 2. The top 30 nodes were determined and could be regarded as opinion leaders based on degree, closeness, and betweenness by using UCINET software, and the results are shown in Table 1.

As shown in Table 1, it can be found that these top 30 nodes have little difference except in order, which supports the evidence to conclude that there is a certain reliability when the method of centrality mentioned in Section 2 was used to identify the corresponding opinion leaders. Therefore, we employed this approach in the subsequent simulation experiment.

3.3. Simulation Results. In general, individual's opinion $\left(x_{0}\right)$ in the initial group obeys random and uniform distribution and $x_{0} \in[-1,+1]$. The initial size of opinion leaders is equal to 20 , and the default method of identification is degree centrality. The proposed model can be used to examine a population of $n$ agents in which a mainstream opinion is in favor of the event in question (i.e., agents in support of the event are the supporters, and the rests are opponents and neutrals). The proportion of the supporters is $P_{s}$, and the rates of the opponents and neutrals are $P_{o}$ and $P_{n}$; $P_{s}+P_{o}+P_{n}=1$. It is assumed that the default values of $P_{s}, P_{o}$, and $P_{n}$ are $80 \%, 15 \%$, and $5 \%$, respectively, and then we can generate the values about opinions for the corresponding groups. For the sake of difference of influence between different groups, we set the influence weight of opinion leaders $\theta=0.8$ and that of ordinary groups $1-\theta=0.2$. Additionally, the default parameters of the simulations tested in the natural reversal function are summarized in Table 2. These parameters are applied throughout all the simulations discussed in this study unless explicitly specified otherwise.

Based on these, we studied the impact of natural reversal parameter $\alpha(t)$ on the evolution of public opinion, which 
TABLE 1: The opinion leaders identified based on three indexes of centrality.

\begin{tabular}{|c|c|c|c|}
\hline No. of nodes & Degree & Closeness & Betweenness \\
\hline 1 & 5 & 5 & 5 \\
\hline 2 & 3 & 3 & 3 \\
\hline 3 & 1 & 2 & 1 \\
\hline 4 & 2 & 1 & 2 \\
\hline 5 & 20 & 20 & 20 \\
\hline 6 & 10 & 4 & 10 \\
\hline 7 & 6 & 6 & 6 \\
\hline 8 & 12 & 10 & 12 \\
\hline 9 & 25 & 12 & 15 \\
\hline 10 & 15 & 9 & 9 \\
\hline 11 & 11 & 11 & 11 \\
\hline 12 & 14 & 15 & 25 \\
\hline 13 & 4 & 37 & 14 \\
\hline 14 & 9 & 8 & 4 \\
\hline 15 & 42 & 14 & 19 \\
\hline 16 & 19 & 19 & 22 \\
\hline 17 & 110 & 63 & 42 \\
\hline 18 & 22 & 32 & 110 \\
\hline 19 & 55 & 27 & 136 \\
\hline 20 & 51 & 22 & 32 \\
\hline 21 & 136 & 26 & 55 \\
\hline 22 & 60 & 7 & 41 \\
\hline 23 & 47 & 68 & 39 \\
\hline 24 & 38 & 39 & 51 \\
\hline 25 & 32 & 42 & 26 \\
\hline 26 & 26 & 16 & 37 \\
\hline 27 & 114 & 25 & 60 \\
\hline 28 & 103 & 17 & 103 \\
\hline 29 & 41 & 136 & 17 \\
\hline 30 & 39 & 34 & 38 \\
\hline
\end{tabular}

Note. No. of nodes refer to the rank in which the centrality is identified from the highest to lowest based on the three kinds of centrality. For example, 1 means the node with the highest centrality, and numbers represent different nodes, such as 5 is node 5 .

TABLE 2: Default simulation parameters.

\begin{tabular}{lc}
\hline Parameter & Value \\
\hline Neighbor type (network topology) & Scale-free network \\
Total population size & $n=1000$ \\
The proportion of the supporters & $P_{s}=80 \%$ \\
The proportion of the opponents & $P_{o}=15 \%$ \\
The proportion of the neutrals & $P_{n}=5 \%$ \\
The influence weight of opinion leaders & $\theta=0.8$ \\
The influence weight of ordinary groups & $1-\theta=0.2$ \\
\hline Identification method of opinion leader & Degree centrality \\
The rate of opinion leader & $R_{m}=2 \%$ \\
Confidence threshold of ordinary agent & $\varepsilon_{i}=0.25$ \\
Confidence threshold of opinion leader & $\eta_{i}=1$ \\
Reversal impact exponents & $\mu=4$ \\
Evolution rate & $\beta=3$ \\
Opinion decay time & $\gamma=10$ \\
Maximum simulation steps & $t=100$ \\
\hline
\end{tabular}

examines the possibility of individuals' own factors and their interactions on the opinion evolution with no interactions. Due to three categories of agents (i.e., supporters, opponents, and neutrals) in our simulations, two simulation cases were taken into account in order to facilitate the analysis of opinion interactions and compare the influence differences. The first case is that the parameter $\alpha(t)$ is introduced for the three types of agents, and this means all individuals' opinions are not only affected by the external interaction with neighboring individuals can be but also updated naturally based on the intrinsic influence of their own factors. Actually, Figures 2 and 3 depict the simulation results of the proposed HK model.

Specifically, Figure 2 represents the opinions of individuals varying with time during the whole evolutionary cycle, in which the red, blue, and black curves depict individuals holding positive, neutral, and negative opinions moving forward in tandem as public opinion reverses, respectively. As shown in Figure 2, it appears as though three types of individuals have reached a compromise, and their opinions of microindividuals converge to the same and show the clear reversal process.

Figure 3 shows the evolution process of the collective opinions over time, where $x(t)$ is the average value of the collective opinions at each time $t$, and this is a tendency. The simulation results indicate there is an obvious natural reversal phenomenon. Due to the large proportion of supporters in the initial stage, the average value of this group's opinions is about 0.4 , which represents a supporting tendency. However, the collective opinions start to reverse and finally reach the stable state with 0 after it continued to increase to 0.7. Accordingly, it can be observed that there is the process of natural reversal dynamics from the individual level and macrolevel. Essentially, as expected, opinion natural reversal involves minor individuals persuading the majority to change opinions in this case. Moreover, in this process, the evolution of an individual opinion is the comprehensive result of interaction with neighbors and natural update according to individual factors themselves.

The second case is that the parameter $\alpha(t)$ is introduced for the agents of supporters only, and this means the opinions of individuals with supportive attitude will be impacted by the interactions with others, as well as the internal factors themselves, while the opinions of other two kinds of participants are just updated according to information interactions with adjacent nodes. The simulation results are shown in Figures 4 and 5.

Similar to Figure 2, the values of supporters' opinions also rise to the peak firstly and then go down to a convergent state. Since the parameter $\alpha(t)$ was not introduced for the opponents and neutrals, a small number of individuals in the social network who are not connected to other nodes will maintain the same opinion values. As can be seen from Figure 4, as expected, this parameter $\alpha$ did indeed have a significant impact on opinion reversal for the supporters and some opponents.

Compared with the results in Figure 5, the tendency of this new experiment for average collective opinions to change with the time is similar to that in Figure 3. As the evolution time increases, the group opinion values increase first and then decrease and eventually reaches the steady state of the system. However, the difference is that the maximum of average opinion value in the system is about 0.55 in Figure 5 and smaller than 0.72 in Figure 3. The possible reason is that when the natural reversal parameter is only introduced for the 


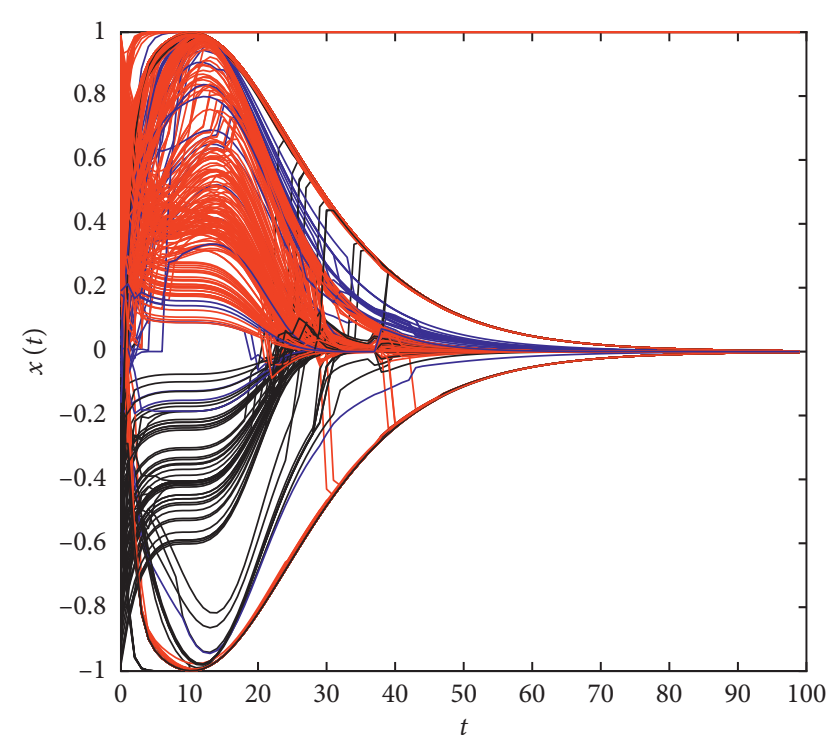

FIGURE 2: Evolution of individual opinions during reversal (I).

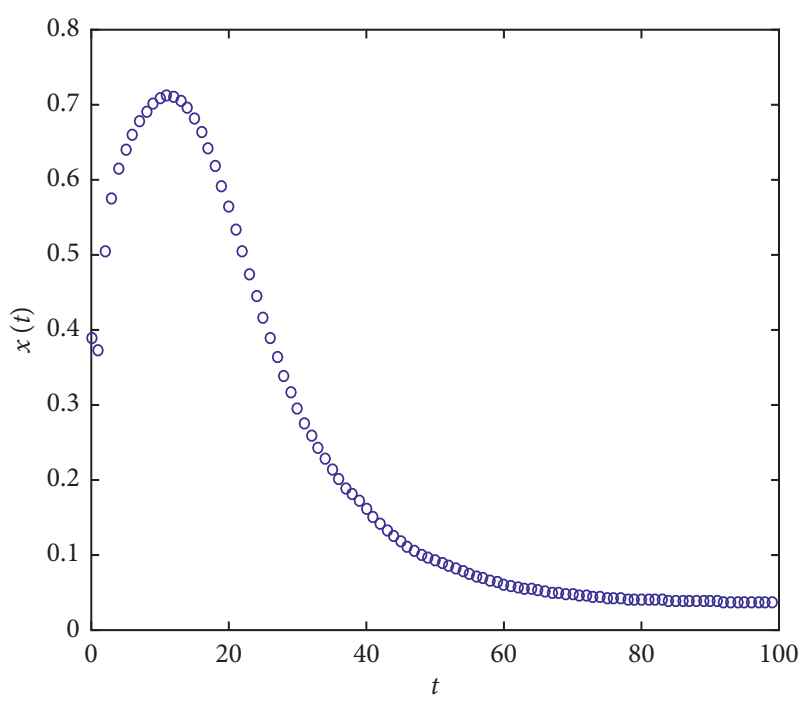

FIGURE 3: Evolution of average collective opinions (I).

supporters, it can found that the opponents' opinions are negative and are greatly different from those of their neighbors, as well as in a state of constant or misconvergence for a long time, as shown in Figure 4, and this is bound to reduce the average opinion value at each time $t$ in the system. This observation evidences the role and effectiveness of natural reversal parameter $\alpha(t)$ in public opinion and posts that $\alpha(t)$ can influence the speed of opinion reversal or stability. Moreover, in the long run, the opinion natural reversal in the system is affected by its own attenuation situation.

Next, in effort to fully elucidate the influence of own attenuation condition, we compare the reversal times with different values about opinion decay time $(\gamma)$ for the average collective opinions of a social system since $\gamma$ is an determinant of the natural reversal parameter $\alpha(t)$. For the sake of simplicity, we change the value of $\gamma$ from 10 to 25 , and the results are shown in Figures 6 and 7.

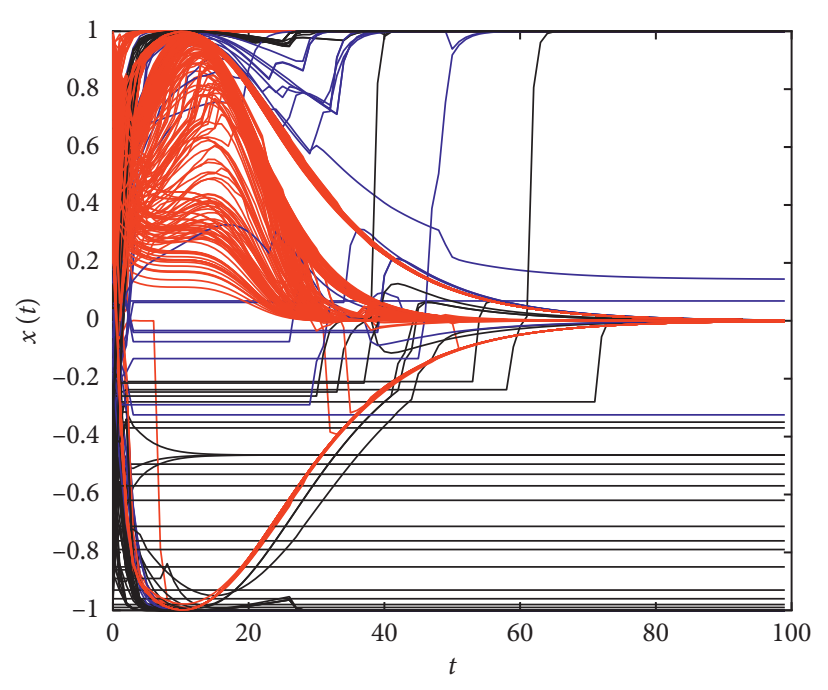

FIgURE 4: Evolution of individual opinions (II).

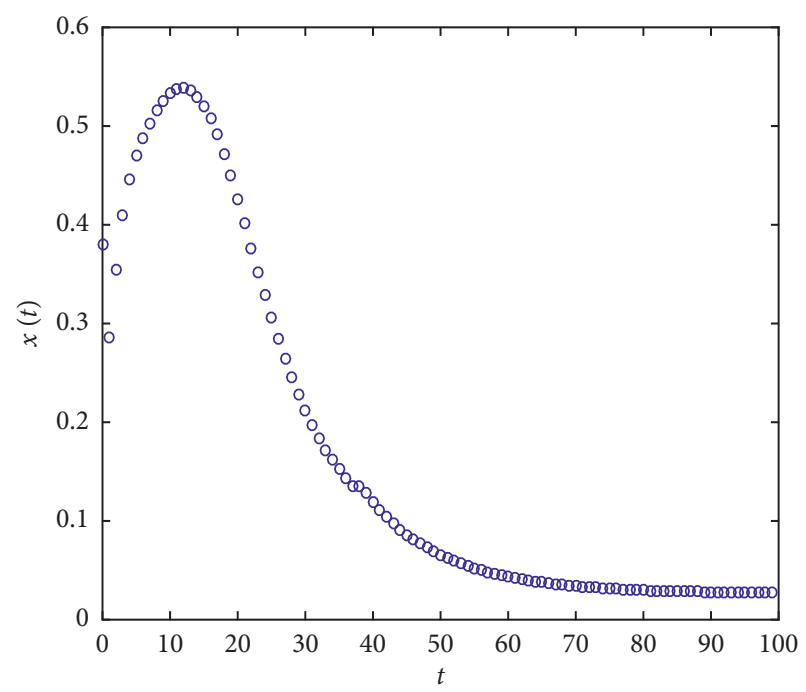

FIGURE 5: Evolution of average collective opinions (II).

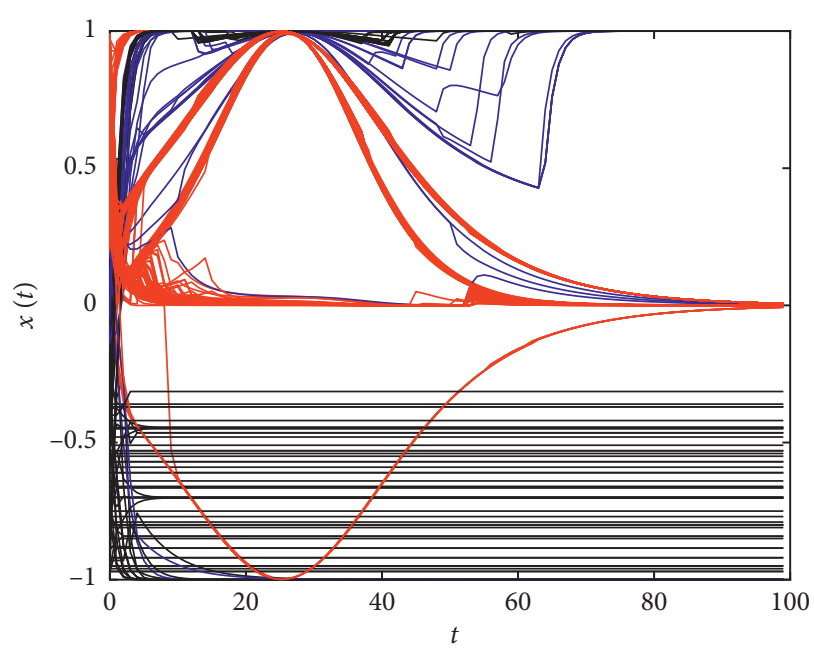

Figure 6: Evolution of individual opinions (III). 


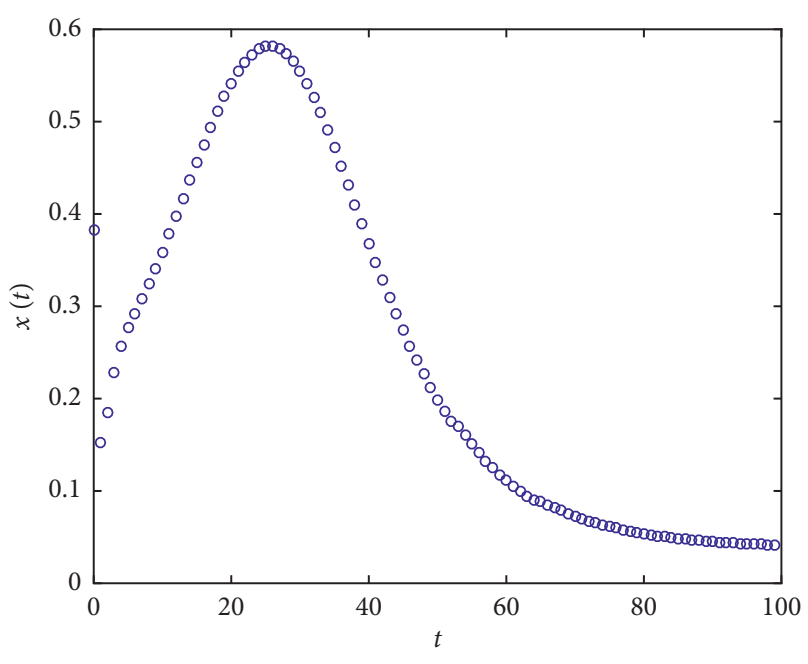

FIGURE 7: Evolution of average collective opinions (III).

Regardless of the parameter values about the opinion decay time $\gamma$, there are similar conclusions for the opinion natural reversal both at the individual and collective levels. However, it is found that its time when the average viewpoint of the system reaches the peak in Figure 7 is obviously lagged behind than that in Figure 5. These indicate that varying influences of different opinion decay time points produced different processes of reversal, which further confirm that the setting of this parameter $\gamma$ in $\alpha(t)$ accords with actual situation.

To sum up, we focus on analyzing the impacts of the natural reversal parameter $\alpha(t)$ and opinion decay time $\gamma$ on the evolution of public opinion. When considering the natural reversal parameter $\alpha(t)$ in both cases above, one can always see that there is indeed an identifiable probability of public opinion natural reversal in a stable state at the end. This further indicates that individual opinions in the social system are impacted by two major aspects to some degree: on the one hand, information interaction or emotional contagion between neighboring nodes may provide individuals with some external pressures or social influences to change their opinions; on the other hand, increasing cognitive levels toward an event may make an individual more prone to identify and justify through his/her own knowledge and experiences, which inherently and significantly affects the individual attitude and thus naturally force to alter his/ her opinions. Besides, regardless of the parameter values about the opinion decay time $\gamma$, the trends of natural reversal for the average collective opinions basically remain the same, while the time reaching the peak is different. Thus, it also suggests that natural reversal parameter $\alpha(t)$ is not a fixed value and will change with some specific parameters, such as $\gamma$. Therefore, we can safely conclude that the proposed model in Section 2 can well simulate and reveal the natural reversal phenomenon and its dynamic process.

\section{Sensitivity Analysis}

In general, individuals do not merely passively interact while exchanging information with others but are constantly influenced by the tendency of group opinion, network structure, and opinion leader. The question is how do we prove these? Thus, the following will conduct the sensitivity analysis with a serial of simulation experiments through the adjustments of corresponding parameters.

4.1. Impact of Proportion of Group Opinions' Tendency on Natural Reversal of Public Opinion. As mentioned above, the group opinions' tendency can be divided into three types, including support, opposition, and neutrality. However, considering that the proportion of neutral participants $\left(P_{n}\right)$ is relatively stable, the default 0.05 was still employed in the proposed model. In addition, their opinions to an event cannot attenuate naturally during a period of time as they do not concern it. This indicates that their opinions will be updated with the interaction between agents, rather than the natural reversal parameter (the same is for the analyses below). Thus, in order to study the impact of proportion of group opinions' tendency on public opinion natural reversal in a social system, we set the rate of individuals with a positive attitude tendency to values of $P_{s} \in[0.5,0.6,0.7,0.8$, 0.9 ] because the majority is over 0.5 in most public opinion models [1], and the other default parameters are shown in Table 2.

Figure 8 presents the simulation results for the opinion evolution in the social system with different values of parameter $P_{s}$. As the value of $P_{s}$ increases from 0.5 to 0.9 , the number of individuals with a higher attitude value increases during evolution. However, a similar public opinion that tended toward reversal in the social system can be observed from the microindividual level, and their steady states always reach consensus, which is consistent with the results in Section 3. This indicates that varying influences of different proportions of group opinions' tendency did not produce obviously different reversal processes of individuals' attitudes. However, the convergence state of individuals initially with opposed attitude was different from the other two groups. Participants with negative opinions can interact with supporters freely; thus, in our simulations, they presented two conditions: one part of individuals insist on their initial attitudes and remain unchanged; another part changed their opinion completely influenced by surrounding individuals especially with positive attitude. Many real-world examples support this observation, such as the evolution of public opinion regarding PX event in China [46].

Figure 9 depicts the simulation results for average collective opinion with different proportion parameters $\left(P_{s}\right)$ and evolutionary time $(t)$. As observed, the larger the proportion of supporters $\left(P_{s}\right)$, the higher the corresponding average collective opinions at each time $t$. Meanwhile, the increase in $P_{s}$ makes the views more convergent consistently. By contrast, when $P_{s}=0.8$ and 0.9 , the reversal of opinions is evident, while opinion did not reverse and the average attitude of the system continuously decay to the steady state when $P_{s}=0.5$, and this is because individuals with negative opinions are difficult to persuade the members with positive opinions to change their original opinions if the portions of these two populations are relatively close. 


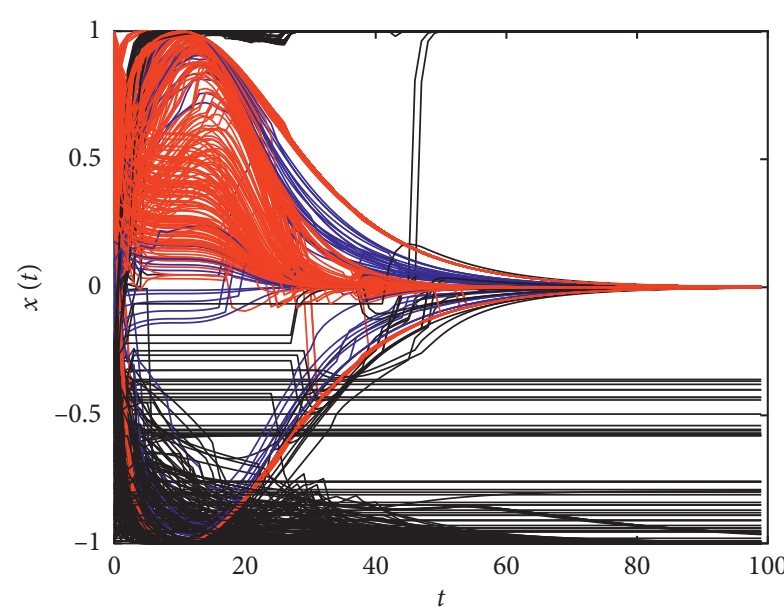

(a)

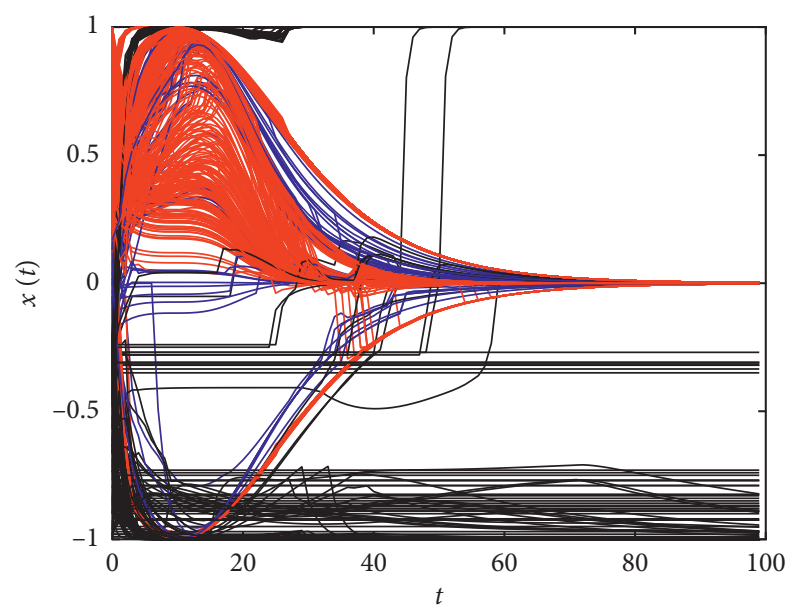

(c)

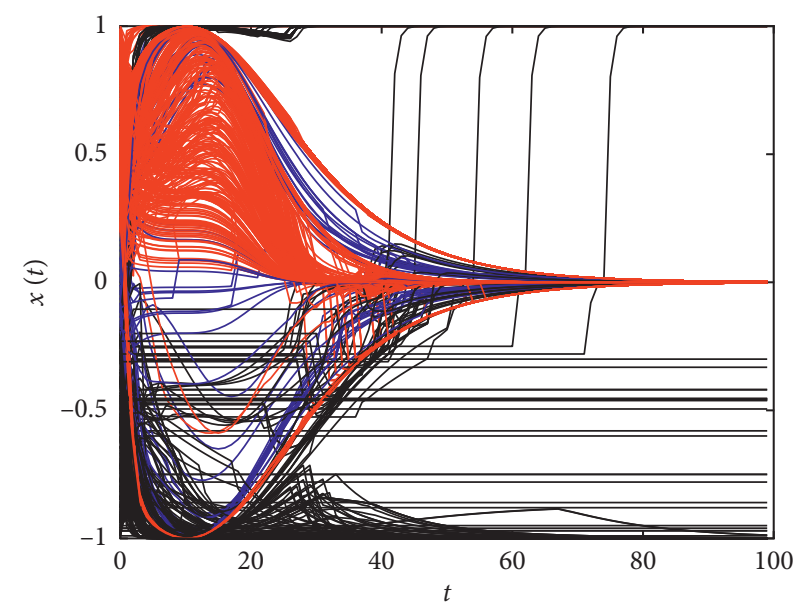

(b)

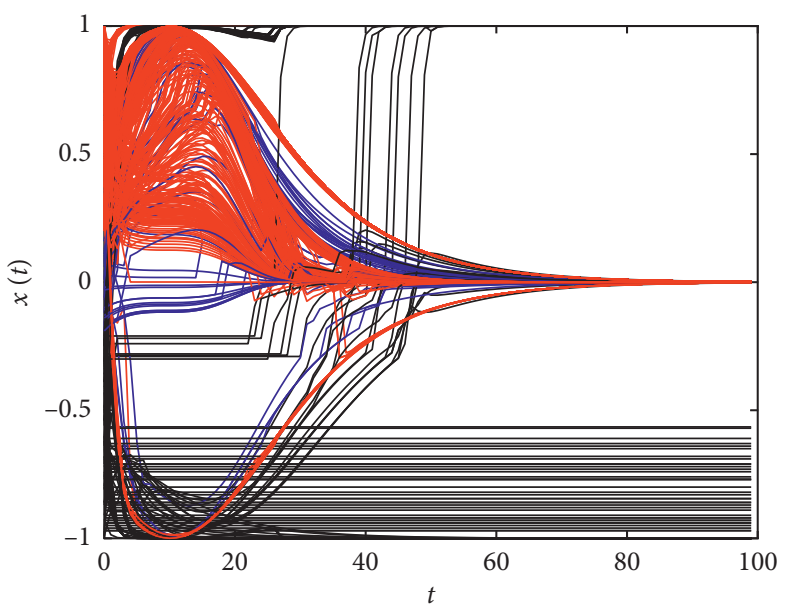

(d)

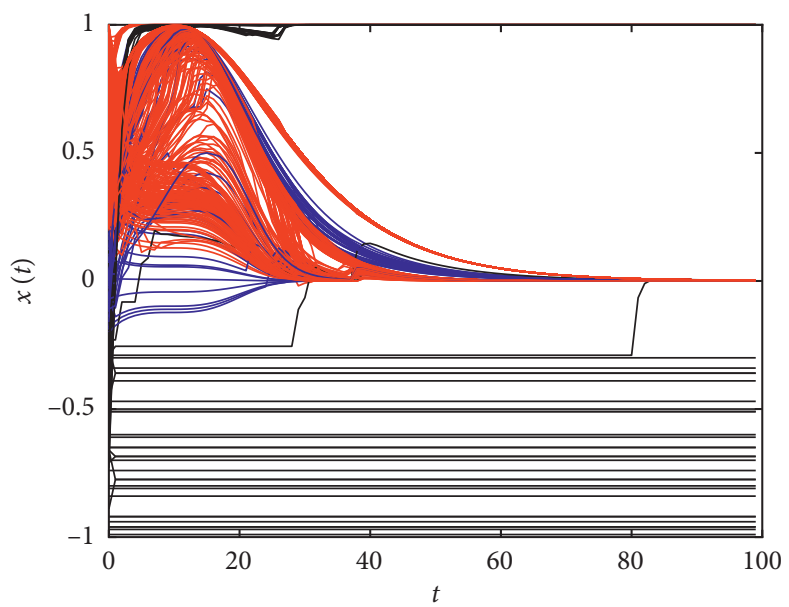

(e)

FIGURE 8: Impact of the proportion of group opinions' tendency on individual opinion reversal: (a) $P_{s}=50 \%, P_{o}=45 \%$, and $P_{n}=5 \%$; (b) $P_{s}=60 \%, P_{o}=35 \%$, and $P_{n}=5 \%$; (c) $P_{s}=70 \%, P_{o}=25 \%$, and $P_{n}=5 \%$; (d) $P_{s}=80 \%, P_{o}=15 \%$, and $P_{n}=5 \%$; (e) $P_{s}=90 \%, P_{o}=5 \%$, and $P_{n}=$ $5 \%$.

As analyzed above, we can conclude that there is a threshold for the proportion parameter $\left(P_{s}\right)$ during the opinion natural reversal. In general, only when $P_{s}$ is greater than the critical value (in our case about 0.5 ), group opinions will represent a natural reversal phenomenon. To some degree, this finding is fundamentally consistent with the 


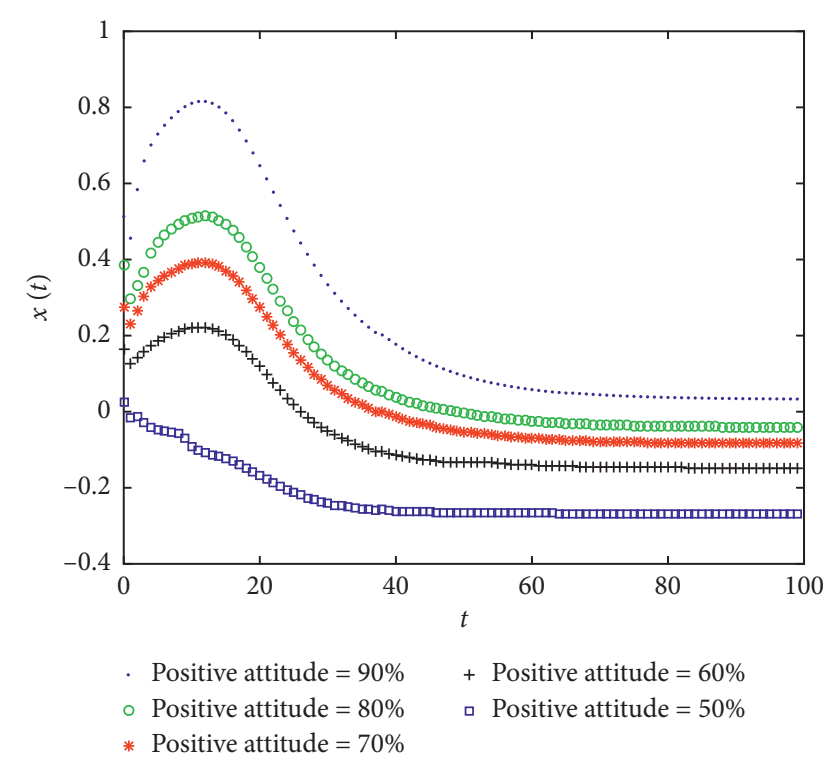

FIGURE 9: Impact of the proportion of group opinions' tendency on average collective opinions.

results in the field of organizational communication researchers who argued that that when an opinion is held by more than $50 \%$ of the sampled individuals [47], it can be regarded as a mainstream idea and significantly affects and thus alters the individual opinions of others. Additionally, it is worth noting that although varying influences of different rates of supporters or opponents did not affect the opinion natural reversal of individuals holding supportive attitude from the microperspective, parameter values can impact the reversal process of the group from the macroperspective. As the public reacts to a certain incident, imbalanced diffusion of information, the presence of cognitive bias of individuals, and varying effects of different interaction intensities caused the different reversal results for the individuals and the groups. Thus, if the minority intends to reverse the public opinion, a high change ratio of opponents may leave them unable to do so.

4.2. Impact of Network Topology on Natural Reversal of Public Opinion. To observe the influence of neighbor type on opinion natural reversal, the default parameters shown in Table 2 are applied to the proposed model to test in three different topologies, and the simulation results are presented in Figures 10 and 11.

It can be seen from Figure 10 that the influence of scalefree network on individual's opinion diffusion is similar to that of small-world network, while the difference is very clear under the random network topology. In the random network, as the time of evolution increases, the steady state of the social system always moves toward polarization and convergence, respectively, indicating the random network does indeed play a significant role in opinion natural reversal. This conclusion is basically consistent with the previous studies. The remaining difference is mainly due to the fact that the proposed model with random network topology takes into account the relatively sufficient interactions among the individuals, and the opinions can be effectively diffused. It is believed that this is one of the reasons why most traditional models of opinion dynamics are built on the basis of random network.

Figure 11 depicts the impact of network topology on average collective opinion. Although the value of average collective opinion in a random network at time $t$ is larger than that of the other two network topological structures, their curve shapes are basically similar, indicating that the network topology fails to play essential influence on the average opinions of the groups. However, by contrast, the opinion's diffusion in the scale-free network is the least sufficient due to few connections between nodes in this topology.

Next, in order to verify the effectiveness of network structure on opinion reversal, we compare the average collective opinions with different proportions of group opinions' tendency for the three network topologies of a social system. Four scenarios of initial groups with different proportions were selected for comparison.

Figure 12 shows the same situation or rule when simulation scenario was divided: the average attitude in a random network is still the highest, while that in a scale-free network is the lowest. Meanwhile, the higher the proportion of supporters, the higher the average level of collective attitudes. Additionally, as the proportion of supporters $\left(P_{s}\right)$ increases from 0.5 to 0.9 , the opinions in the social system reverse more significantly for the three network topologies. Besides, according to the trends in Figure 12(d), we can infer that public opinion might not reverse if $P_{s}<50 \%$ under the scale-free network, which is consistent with the result in Section 4.1.

\subsection{Impact of Opinion Leader on Natural Reversal of Public} Opinion. As mentioned in Section 3.2, it can be found that the nodes of opinion leaders determined by three types of centrality are the same basically, but there are some differences in the order of identification. Therefore, to explore the effect of opinion leader on natural reversal of public opinion, we firstly analyze the influence of three identification approaches on the opinion reversal. In our study, we simulated successively based on degree centrality (namely centrality), closeness centrality, and betweenness centrality, and the other parameters still used the default values. The results are shown in Figures 13 and 14.

On the whole, the results in Figure 13 show that the reversal trend and evolutionary process of individual opinions based on three centralities are similar, it is mainly because there is little difference in the opinion leaders determined by these three methods, and the proportion of $2 \%$ may be too small to have a significant effect.

However, it can be seen from Figure 14 that although there is little difference in the identification of opinion leaders, the average value of collective opinions based on the degree centrality is the highest at each time $t$. This indicates that the identification order of opinion leaders could determine the degree of opinion's interaction. In general, the node connected with more neighbors can directly control attitude 


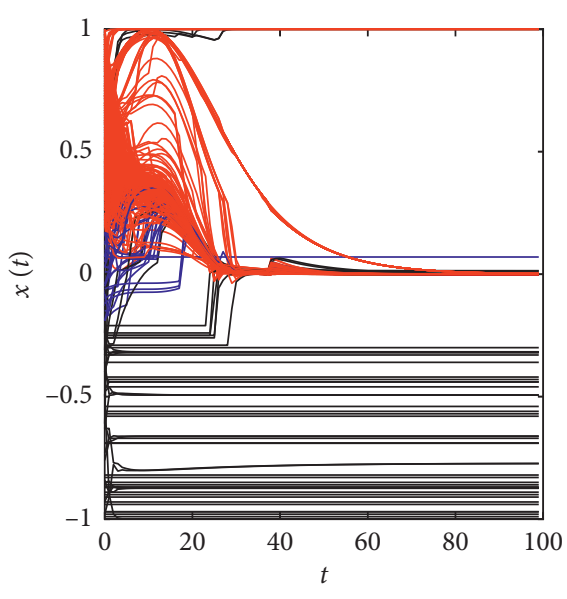

(a)

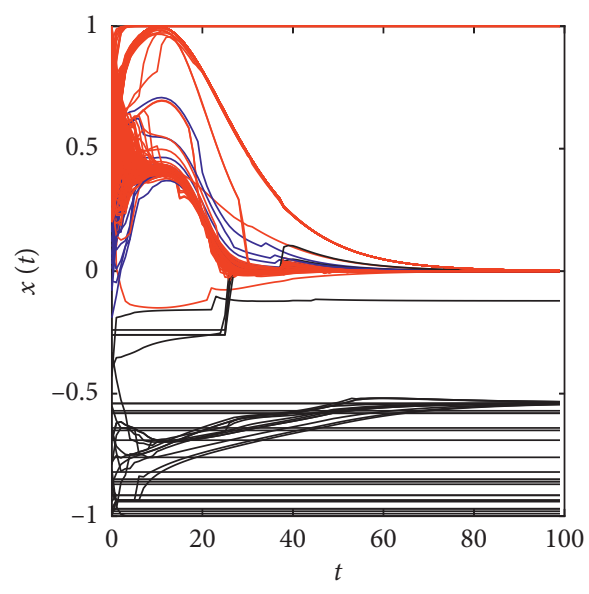

(b)

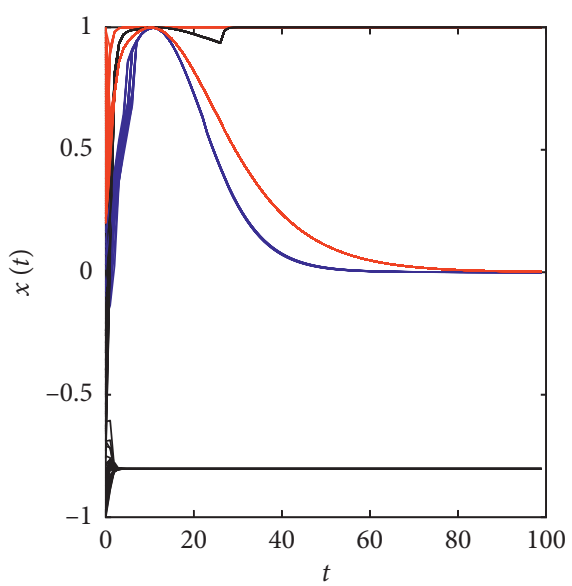

(c)

FIGURE 10: Impact of network topology on individual opinion reversal: (a) scale-free network; (b) small-world network; (c) random network.

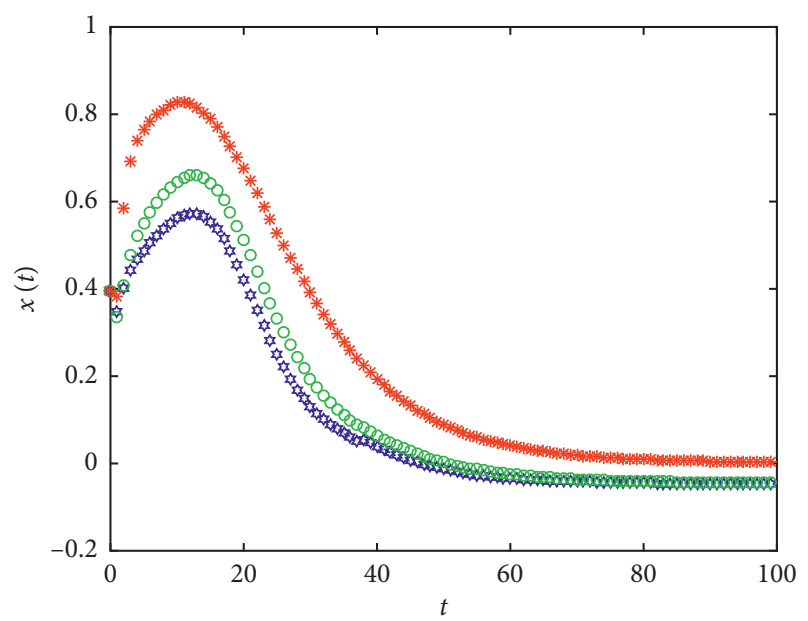

$$
\begin{aligned}
& \text { * Scale free } \\
& \text { - Small world } \\
& \text { * ER }
\end{aligned}
$$

FIGURE 11: Impact of network topology on evolution of average collective opinions. interaction and sufficiently spread, and thus, the opinion leaders identified with the approach of degree centrality will play an important role in the viewpoint diffusion.

Furthermore, we compared opinion evolutions for four initial conditions with different proportions of supporters. Although the ratios of three groups are diverse, as shown in Figure 15, public opinions in a social system always reverse naturally under the three kinds of centralities. However, as the proportion of supporters $\left(P_{s}\right)$ increases, all the average values of attitude increase in three identification methods of opinion leader. Accordingly, it can conclude that initial attitude tendency of the group fails to obviously change the effect of these three identification methods of opinion leaders on opinion natural reversal. Besides, according to the trends in Figure 15(d), it is possible that public opinion might not reverse when $P_{s}$ decreases to smaller than 0.5 . As a result, both identification of opinion leader and initial attitude tendency of the group can play important roles in the opinion natural reversal.

Secondly, even if the neighboring node $j$ is an opinion leader determined by the three approaches, its impact on the 


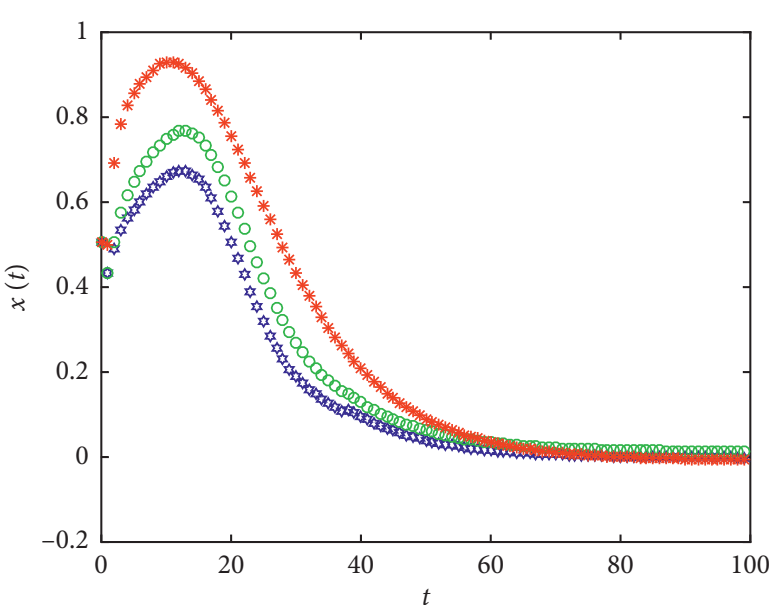

* Scale free

- Small world

* ER

(a)

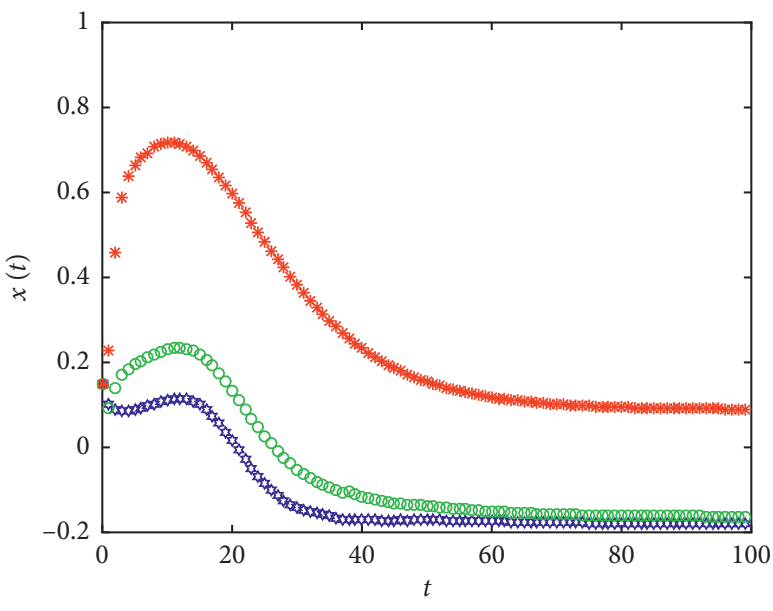

- Scale free

- Small world

* ER

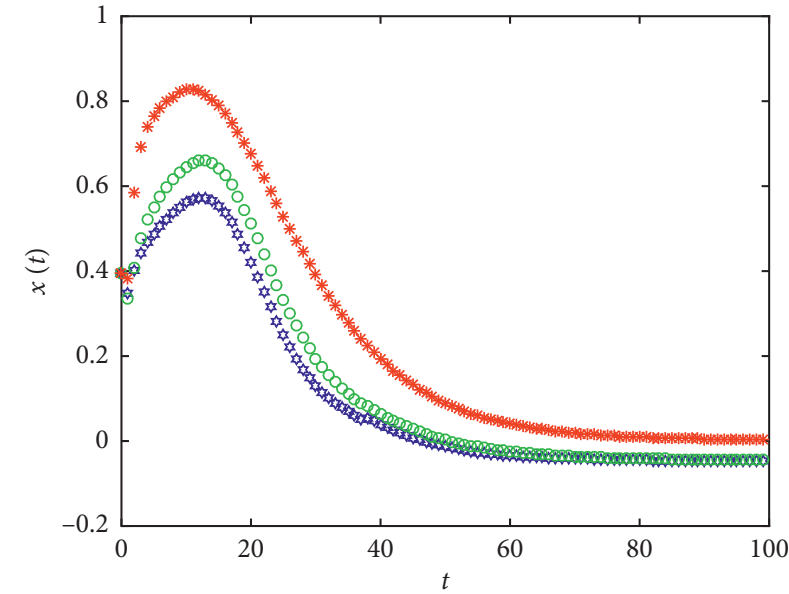

* Scale free

- Small world

* ER

(b)

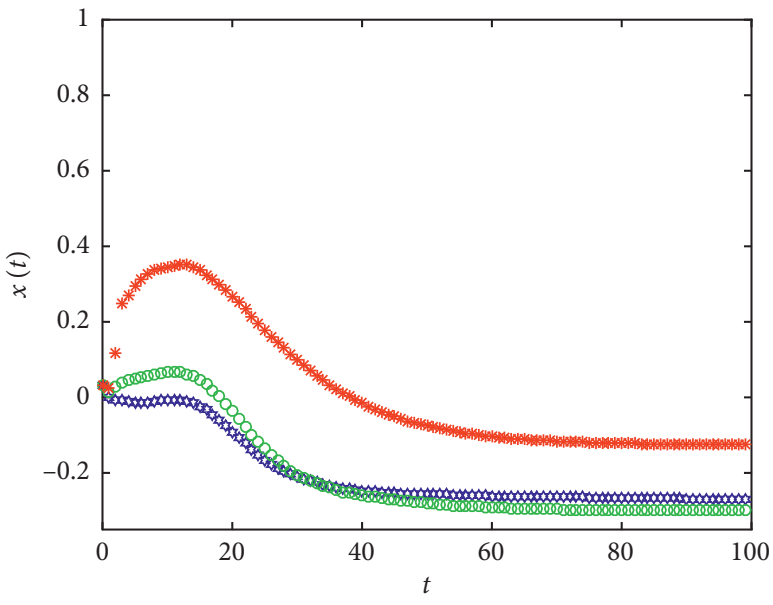

* Scale free

- Small world

* ER

(d)

Figure 12: Evolution of average collective opinions under three topologies: (a) $P_{s}=90 \%, P_{o}=5 \%$, and $P_{n}=5 \%$; (b) $P_{s}=80 \%, P_{o}=15 \%$, and $P_{n}=5 \%$; (c) $P_{s}=70 \%, P_{o}=25 \%$, and $P_{n}=5 \%$; (d) $P_{s}=50 \%, P_{o}=45 \%$, and $P_{n}=5 \%$.

evolution of individual opinion may be different due to his/ her different influence powers. To unfold this impact, parameter $\theta$ is employed to measure the influence power of opinion leader in our proposed model. Due to the consideration of simplicity, we set influence power parameter $\theta=0,0.2,0.4,0.6,0.8$, and 1.0 to represent individuals affecting varying degrees by their influence powers of opinion leaders. The results are shown in Figure 16.

Figure 16 depicts that the smaller the influence power of an opinion leader, the smaller the amplitude of individual opinions at the same time $t$, which means the amplitude of individual opinions would shift with the influence weight $\theta$. However, we also found that when $\theta$ is relatively small, the values of individual attitudes will be more likely to reach a larger level at the early stage. This indicates that when the influence of an opinion leader is insufficient, individuals are much easier to determine their opinions' tendency at the time $t+1$ and to achieve the consensus. As far as we know, the influence power of opinion leader is dependent on one's authority, credibility, and some other related aspects, so an opinion leader is difficult to exert effective influence on the opinion followers if the influence power is too small or in a society with low trust.

Figure 17 denotes the curves of average collective opinions based on different influence weights of opinion leader. When $\theta$ is not high (e.g., $\theta=0$ denotes there is no opinion leader), despite the opinions $x(t)$ decay over time, there is a greater possibility that opinion natural reversal will not occur. Correspondingly, when it comes to the joint influences of opinion leaders and ordinary agents (e.g., 


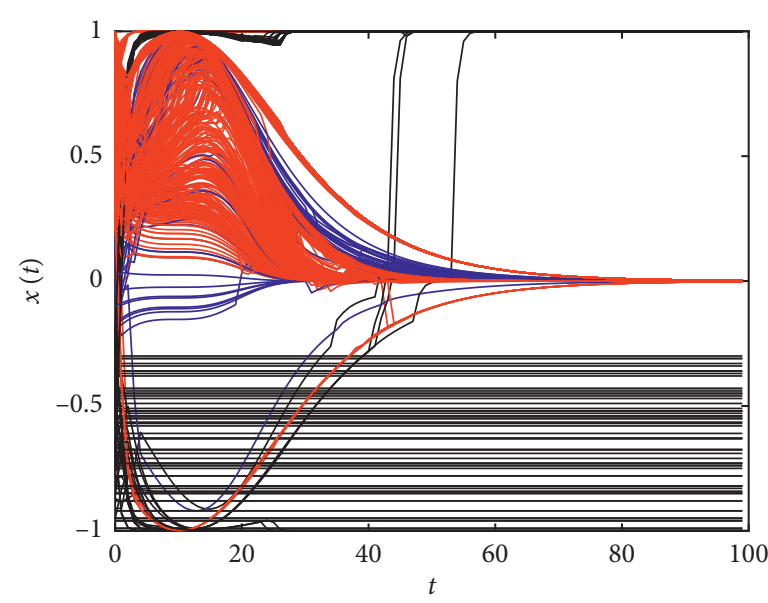

(a)

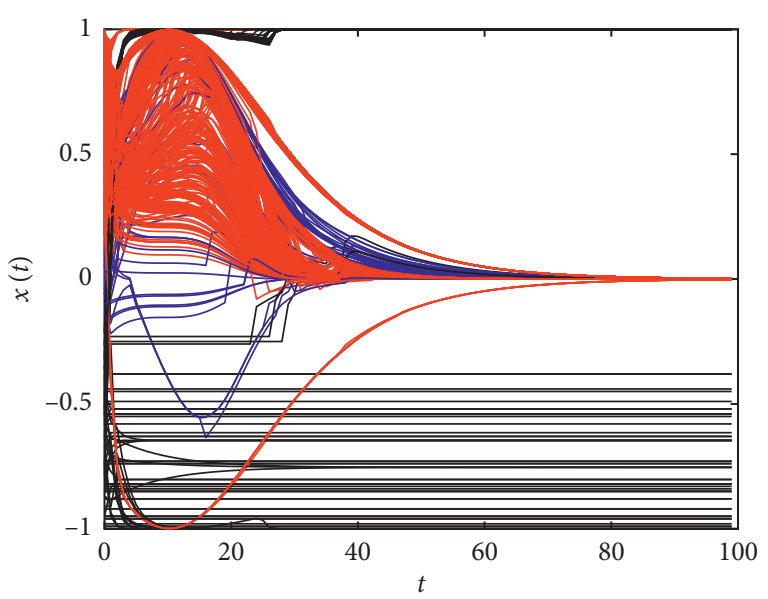

(b)

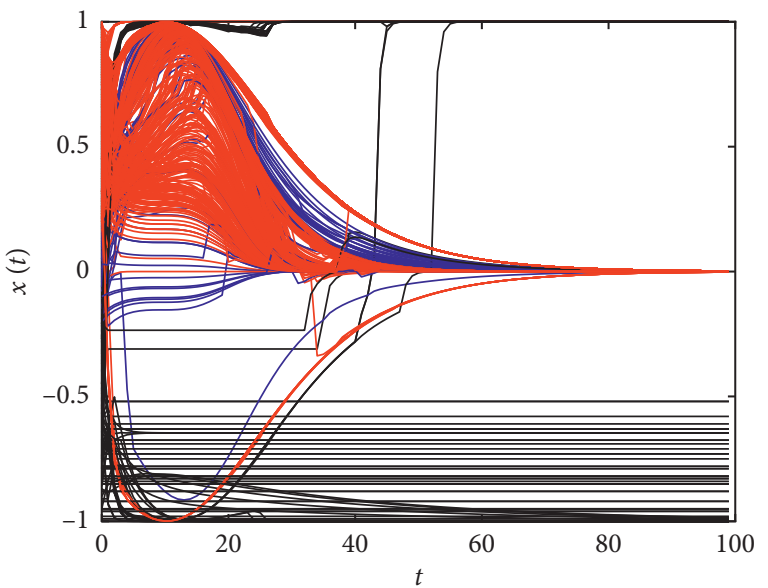

(c)

FIGURE 13: Impact of centrality approach on individual opinion reversal: (a) centrality; (b) closeness centrality; (c) betweenness centrality.

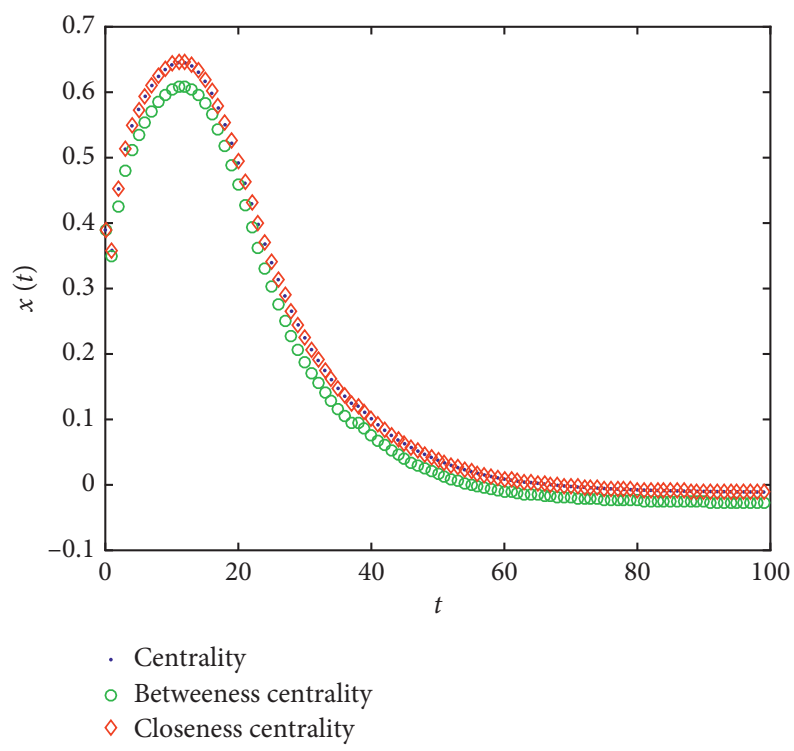

FIGURE 14: Evolution of average collective opinions based on three centralities. 


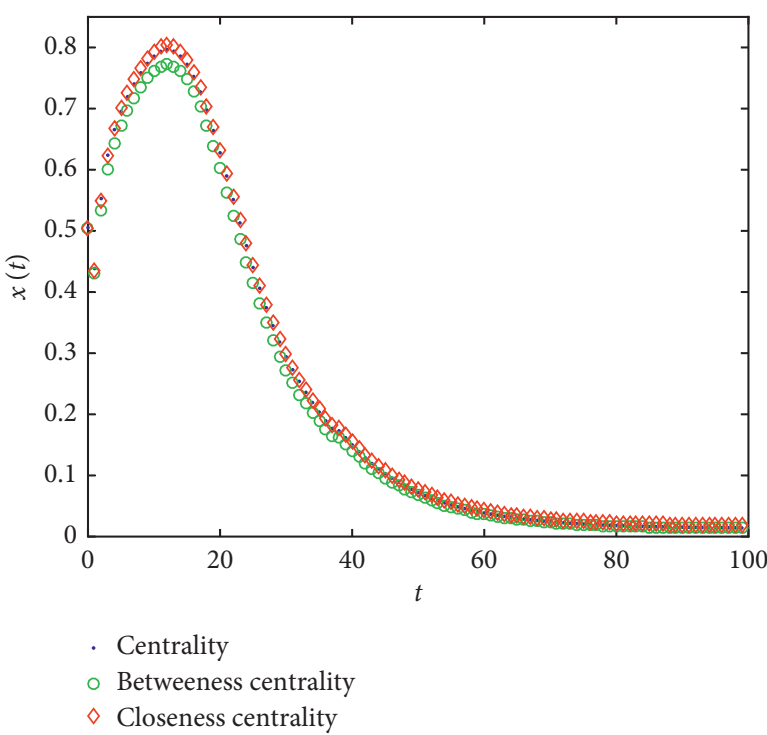

(a)

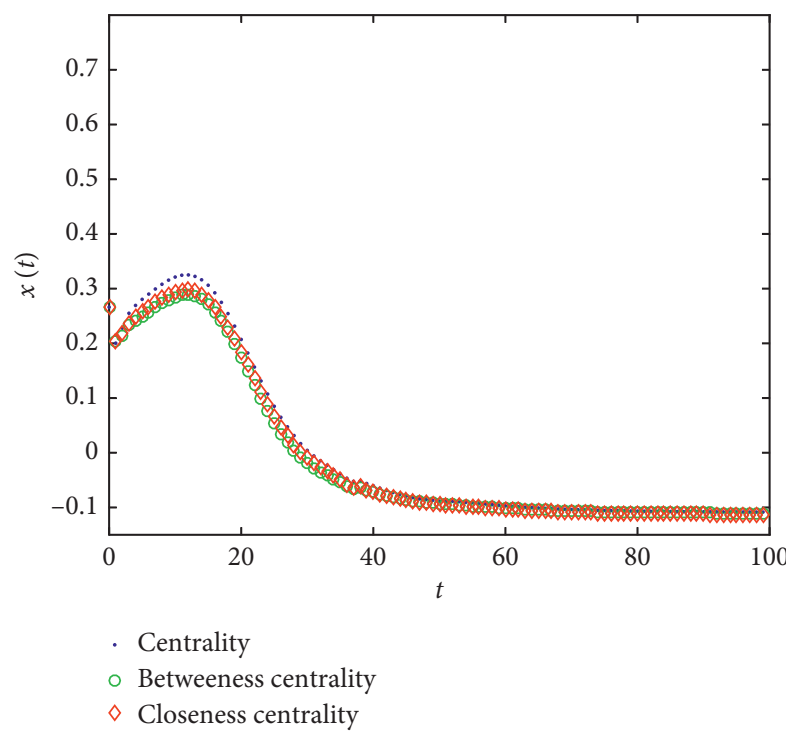

(c)

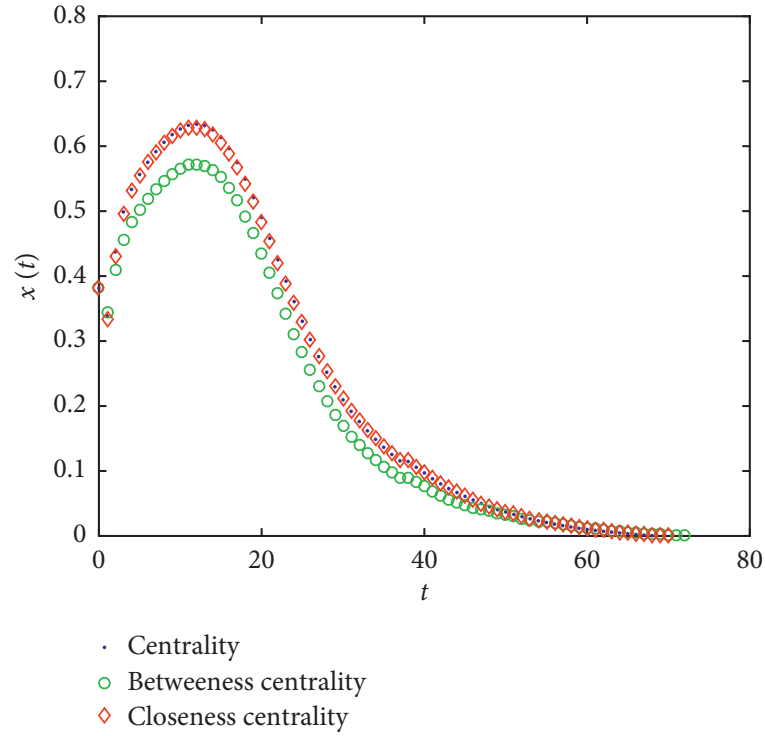

(b)

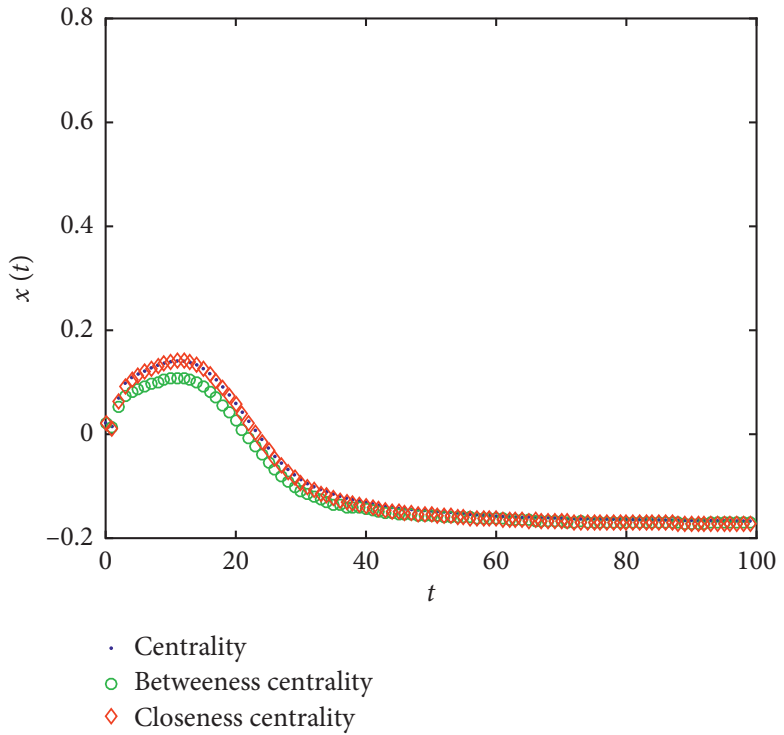

(d)

Figure 15: Evolution of average collective opinions based on three centralities: (a) supporters: 90\%, opponents: 5\%, and neutrals: 5\%; (b) supporters: $80 \%$, opponents: $15 \%$, and neutrals: $5 \%$; (c) supporters: $70 \%$, opponents: $25 \%$, and neutrals: $5 \%$; (d) supporters: $50 \%$, opponents: $45 \%$, and neutrals: $5 \%$.

$0<\theta<1.0$ ), the results show that the average value of group attitudes does not increase as $\theta$ increase, while it is the highest when $\theta$ is about 0.5 . However, the influence of opinion leader's attitude is only taken into account (e.g., $\theta=1.0$ ), and the average value of the collective opinions will be not the highest instead. This is because although the diffusion of attitudes is significantly affected by the opinion leaders, any one individual agent interacts with a large number of common agents who account for the vast majority in the population size, while the opinion leaders are just the minor group. Therefore, the impact of the influence weight of opinion leaders on the group views is not a simple positive linear relationship, but there is a peak from $\theta=0$ to 1. Accordingly, we can assert that our simulation results for the impact of $\theta$ on the opinion natural reversal is reliable in a social system.

Taken together, as discussed above, the roles of proportion of group opinions' tendency, network topology, and opinion leader in the natural reversal of public opinion have been proved. What is important, regardless of the types of 


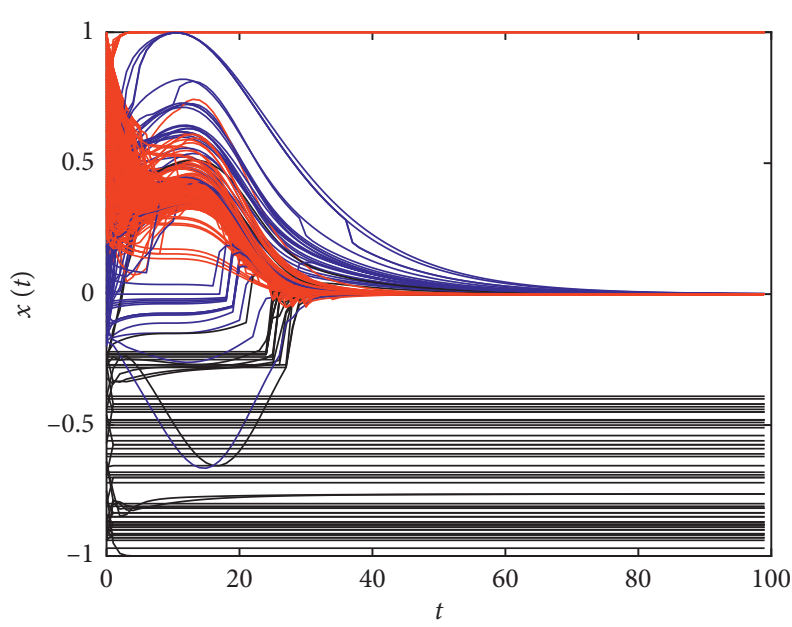

(a)

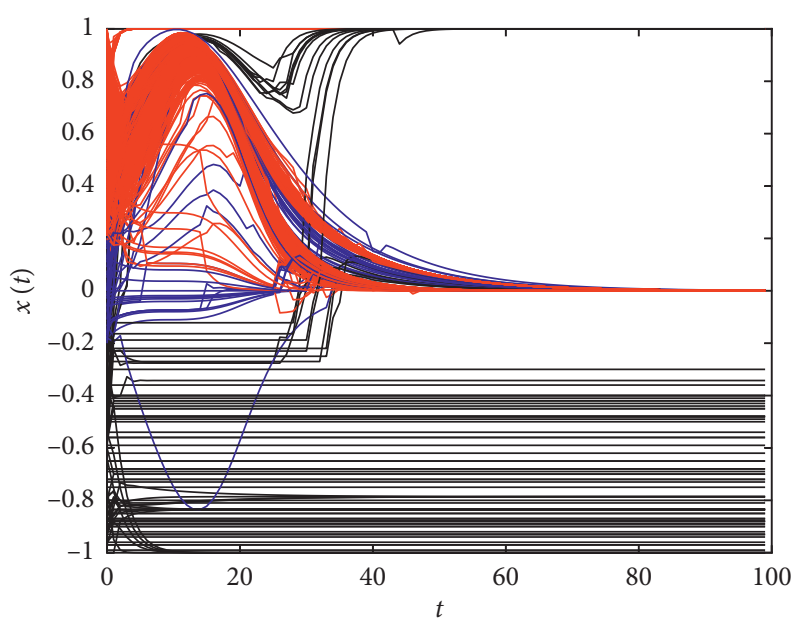

(c)

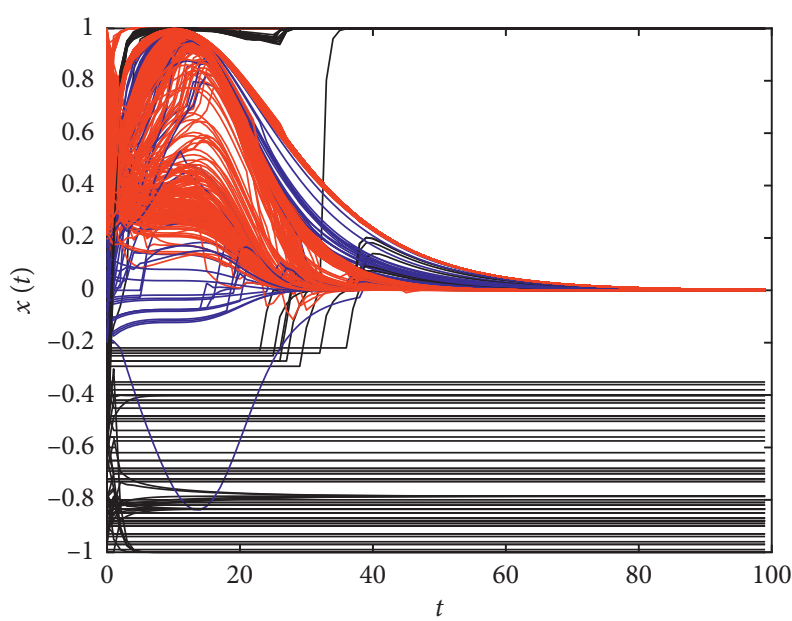

(e)

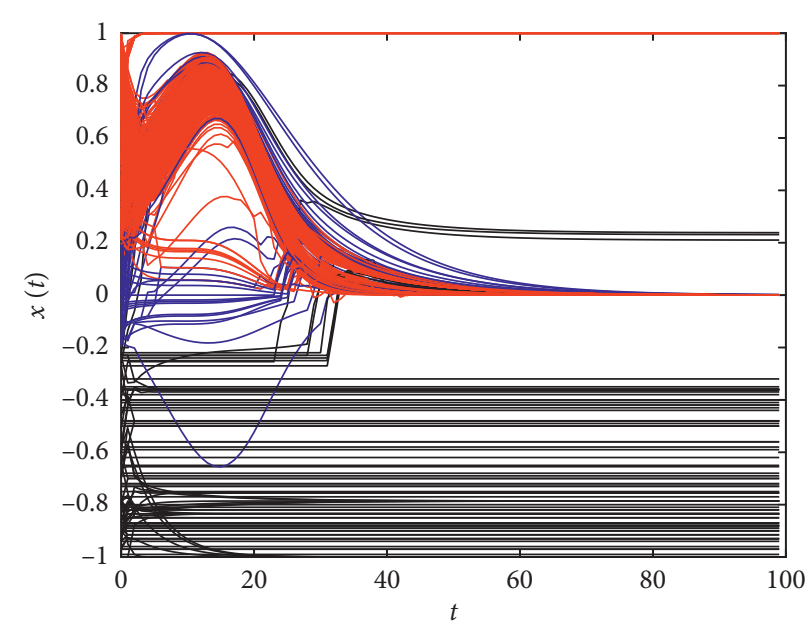

(b)

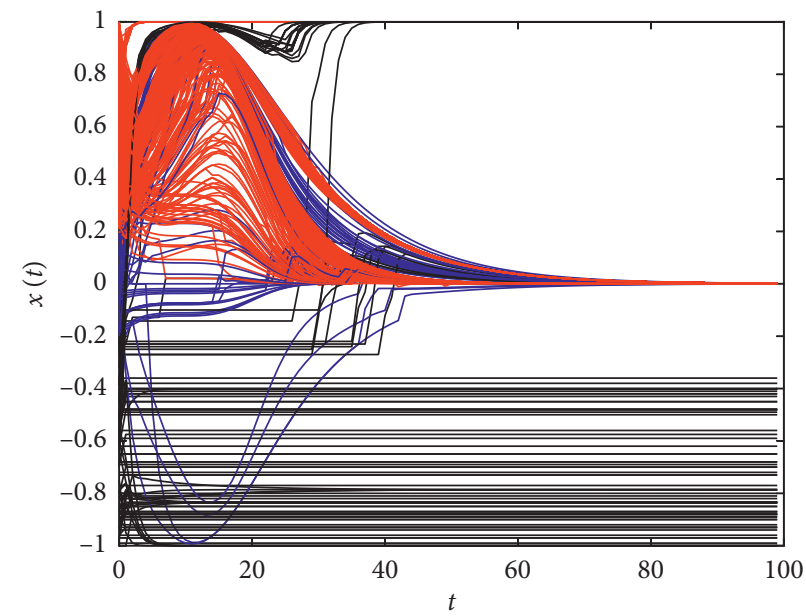

(d)

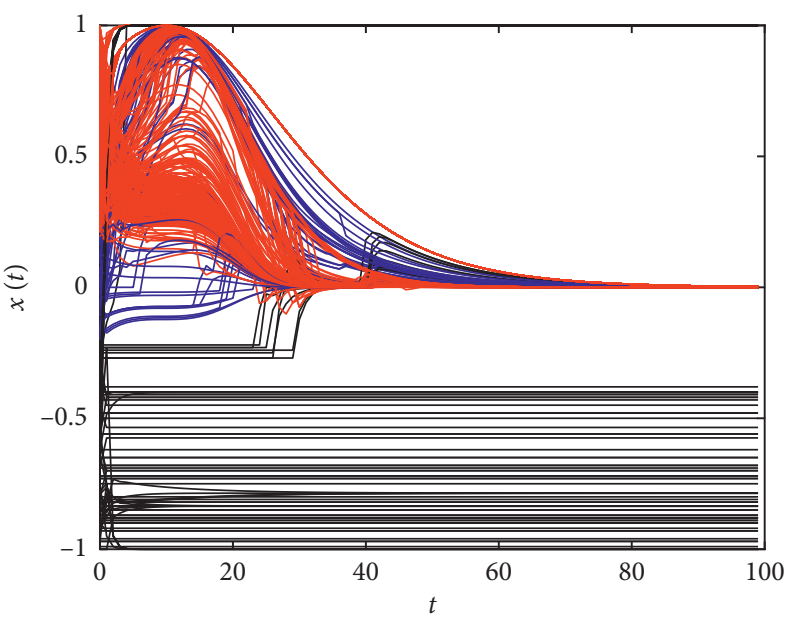

(f)

FIGURE 16: Impact of the influence power of opinion leaders on individual opinion reversal: (a) $\theta=0$; (b) $\theta=0.2$; (c) $\theta=0.4$; (d) $\theta=0.6$; (e) $\theta=0.8$; (f) $\theta=1.0$.

network topologies and identification methods of opinion leader, we can argue that the natural reversal of public opinion always occurs as long as the proportion of individuals with initial supportive attitude is greater than 0.5 , which is consistent with the opinion reversal fact of minor individuals persuading the majority to change opinions. 


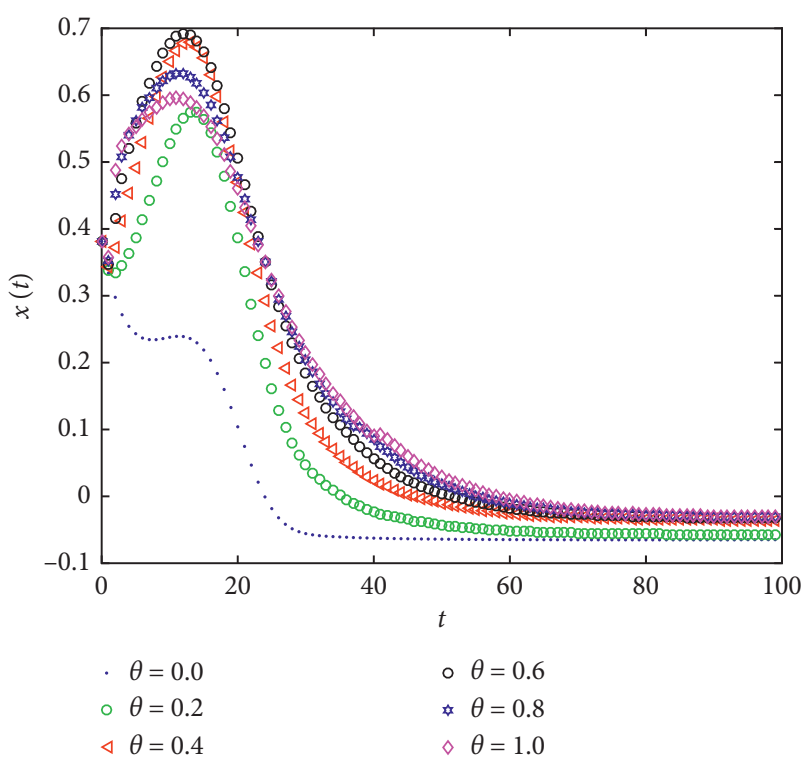

Figure 17: Evolution of average collective opinions based on different influence powers of opinion leader.

\section{Conclusion and Discussion}

In this study, the influence of natural reversal parameter and opinion leaders was considered in the context of opinion dynamics, and a public opinion evolution model based on the HK model was proposed and simulated. Some illuminating findings are summarized as following:

(1) Natural reversal parameter $\alpha(t)$ plays an important role in the dynamic evolution of opinion, which indicates that individuals involved in the evolution of public opinion not only vary their attitudes based on the opinions of their different kinds of neighbors by utilizing an influence weight (e.g., external peer influence) but also refer to their own factors to change naturally with no interactions (e.g., internal experience judgment). In addition, the natural reversal parameter $\alpha(t)$ is not a fixed value, but a monotone increasing function determined by specific factors. Therefore, compared to the classical HK model, the simulation results revealed that our novel model is much closer to the actual situation on virtual Internet world and can give a more accurate and reasonable description for the process of opinion reversal through online interactions.

(2) In the reversal process of public opinion, both the network topology and opinion leader obviously exert important roles. Specifically, to the individual specific level, the effects of three topologies on the fusion of individual opinions are clearly different. However, these impacts of network structure are not affected by the initial distribution of collective opinion, which indicates that we cannot ignore the importance of natural interactions between the individuals and their neighbors. As well, on the one hand, it can be found that identification methods of opinion leaders based on three types of centralities have similar influences on the evolution process of individual opinions since the opinion leader nodes determined by these methods are basically similar, but the values of group views based on degree centrality is the highest at the same time $t$. This manifests that the opinion leader is central in online public opinion. On the other hand, only when influence power parameter $(\theta)$ of an opinion leader is bigger than a certain value and will opinion natural reversal occur. However, due to the minority of opinion leaders, a large number of ordinary groups will also impact the adoption of individual views, which is consistent with participant's "the herd effect" in reality. Therefore, the influence power parameter $(\theta)$ of an opinion leader is not always positively correlated with the change in group views.

(3) In the evolution of public opinion, the proportion of supporters $\left(P_{s}>0.5\right)$ is larger, and the average collective opinions become higher, which further makes the views more convergent consistently. Thus, the effect of the proportion of group opinions' tendency can speed up the integration of public opinion, as well the trend of natural reversal of group opinions from the macrolevel. This is because when there are the majority of individuals holding supportive attitude, they will play a significant assimilation or contagion role for the agent, which further enhances the fusion of group opinions and more likely retains the consensus opinions in the system. Besides, this finding is also observed under the different network topologies and identifying methods of opinion leader, which further confirms the robust of our model.

We can conclude that our proposed model is an effective extension of the HK model. The prior dynamics model considers the nonlinear feature of public opinion evolution, but it mainly explains the evolution mechanism of the interaction between individuals. However, the evolution of public opinion in the real society is extremely complex, and citizens have more and more ways to obtain and generate information. Thus, when an individual updates his/her opinion, it is no longer only influenced externally by the other individuals but also relies on the natural drive of their own internal cognition. We assert that there is some novelty regarding the natural reversal parameter in our proposed model. Besides, the simulations in this study have examined the relationship between natural reversal of group views and dynamic change in individual opinions, which can contribute to shedding new insights for understanding opinion dynamics more comprehensively. However, admittedly, there are also some limitations. For instance, to simplify the study, the default values of some parameters in our simulations were chosen according to the related works, and more practical cases are needed to test this model in the future. Additionally, the natural reversal parameter is crucial to the opinion evolution, but the limited factors including the time, influence index, evolution rate, and decay time 
were taken into account when it was designed; in the future, some qualitative or quantitative methods can be applied to comprehensively explore the determinants of this parameter, which in turn can more accurately explain the issue of opinion natural reversal process.

\section{Data Availability}

The data used to support the findings of this study are available from the corresponding author upon request.

\section{Conflicts of Interest}

The authors declare that there are no conflicts of interest regarding the publication of this paper.

\section{Acknowledgments}

This work was supported by the National Science and Technology Innovation 2030 Major Project of the Ministry of Science and Technology of China (Grant no. 2018AAA0101200) and the National Natural Science Foundation of China (Grant nos. 71572185, 71874163, and 61540032).

\section{References}

[1] C. Huang, B. Hu, G. Jiang, and R. Yang, "Modeling of agentbased complex network under cyber-violence," Physica A: Statistical Mechanics and Its Applications, vol. 458, pp. 399$411,2016$.

[2] K. Starcke and M. Brand, "Decision making under stress: a selective review," Neuroscience \& Biobehavioral Reviews, vol. 36, no. 4, pp. 1228-1248, 2012.

[3] C. Castellano, S. Fortunato, and V. Loreto, "Statistical physics of social dynamics," Review of Modern Physics, vol. 81, no. 2, pp. 591-646, 2007.

[4] M. H. Degroot, "Reaching a consensus," Journal of the American Statistical Association, vol. 69, no. 345, pp. 118-121, 1974.

[5] G. Albi, L. Pareschi, and M. Zanella, "Opinion dynamics over complex networks: kinetic modelling and numerical methods," Kinetic and Related Models, vol. 10, no. 1, pp. 1-32, 2017.

[6] S. Monica and F. Bergenti, "Opinion dynamics in multi-agent systems: selected analytic models and verifying simulations," Computational and Mathematical Organization Theory, vol. 23, no. 3, pp. 423-450, 2017.

[7] R. Fan, K. Xu, and J. Zhao, "An agent-based model for emotion contagion and competition in online social media," Physica A: Statistical Mechanics and Its Applications, vol. 495, pp. 245-259, 2018.

[8] B. Düring and M.-T. Wolfram, "Opinion dynamics: inhomogeneous Boltzmann-type equations modelling opinion leadership and political segregation," Proceedings of the Royal Society A-Mathematical Physical and Engineering Sciences, vol. 471, no. 2182, 2015.

[9] T. Filatova, P. H. Verburg, D. C. Parker, and C. A. Stannard, "Spatial agent-based models for socio-ecological systems: challenges and prospects," Environmental Modelling \& Software, vol. 45, pp. 1-7, 2013.

[10] S. Galam, Y. Gefen, and Y. Shapir, "Sociophysics: a new approach of sociological collective behaviour. I. mean- behaviour description of a strike," The Journal of Mathematical Sociology, vol. 9, no. 1, pp. 1-13, 1982.

[11] S. Galam and S. Moscovici, "Towards a theory of collective phenomena: consensus and attitude changes in groups," European Journal of Social Psychology, vol. 21, no. 1, pp. 4974, 2010.

[12] K. Sznajd-Weron and J. Sznajd, "Opinion evolution in closed community," International Journal of Modern Physics C, vol. 11, no. 6, pp. 1157-1165, 2000.

[13] S. Galam, "Minority opinion spreading in random geometry," The European Physical Journal B, vol. 25, no. 4, pp. 403-406, 2002.

[14] U. Krause, "A discrete nonlinear and non-autonomous model of consensus formation," in Communications in Difference Equations, S. Elyadi, G. Ladas, and J. Popenda, Eds., pp. 227-236, Gordon and Breach Pub., Amsterdam, Netherlands, 2000.

[15] R. Hegselmann and U. Krause, "Opinion dynamics and bounded confidence: Models, analysis and simulation," Journal of Artificial Societies \& Social Simulation, vol. 5, no. 3, pp. 1-33, 2002.

[16] G. Deffuant, D. Neau, F. Amblard, and G. Weisbuch, "Mixing beliefs among interacting agents," Advances in Complex Systems, vol. 3, no. 4, pp. 87-98, 2000.

[17] G. Weisbuch, G. Deffuant, F. Amblard, and J. P. Nadal, "Meet, discuss, and segregate," Complexity, vol. 7, no. 3, pp. 55-63, 2002.

[18] J. Lorenz, "Continuous opinion dynamics under bounded confidence: a survey," International Journal of Modern Physics C, vol. 18, no. 12, pp. 1819-1838, 2007.

[19] T. Krueger, J. Szwabinski, and T. Weron, "Conformity, anticonformity and polarization of opinions: insights from a mathematical model of opinion dynamics," Entropy, vol. 19, no. 7 , p. 371, 2017.

[20] T. Kobayashi, "Selective exposure," The International Encyclopedia of Political Communication, vol. 3, pp. 1-8, 2016.

[21] F. Gargiulo and S. Huet, "Opinion dynamics in a group-based society," EPL (Europhysics Letters), vol. 91, no. 5, pp. 2067-2076, 2010.

[22] Y. Shang, "Deffuant model with general opinion distributions: first impression and critical confidence bound," Complexity, vol. 19, no. 2, pp. 38-49, 2013.

[23] J. Zhang and Y. Hong, "Opinion evolution analysis for shortrange and long-range Deffuant-Weisbuch models," Physica A: Statistical Mechanics and Its Applications, vol. 392, no. 21, pp. 5289-5297, 2013.

[24] L. Li, A. Scaglione, A. Swami, and Q. Zhao, "Consensus, polarization and clustering of opinions in social networks," IEEE Journal on Selected Areas in Communications, vol. 31, no. 6, pp. 1072-1083, 2013.

[25] Q. Liu and X. Wang, "Social learning with bounded confidence and heterogeneous agents," Physica A: Statistical Mechanics \& Its Applications, vol. 392, no. 10, pp. 2303-2306, 2013.

[26] E. Kurmyshev, H. A. Juárez, and R. A. González-Silva, "Dynamics of bounded confidence opinion in heterogeneous social networks: concord against partial antagonism," Physica A: Statistical Mechanics and Its Applications, vol. 390, no. 16, pp. 2945-2955, 2011.

[27] M. Pineda, R. Toral, and E. Hernández-García, "Diffusing opinions in bounded confidence processes," The European Physical Journal D, vol. 62, no. 1, pp. 109$117,2011$.

[28] X. Chen, X. Zhang, Z. Wu, H. Wang, G. Wang, and W. Li, "Opinion evolution in different social acquaintance 
networks," Chaos: An Interdisciplinary Journal of Nonlinear Science, vol. 27, no. 11, Article ID 113111, 2017.

[29] G. Kou, Y. Zhao, Y. Peng, Y. Shi, and P. Holme, "Multi-level opinion dynamics under bounded confidence," PLoS One, vol. 7, no. 9, Article ID e43507, 2012.

[30] Y. Zhu, Q. A. Wang, W. Li, and X. Cai, "The formation of continuous opinion dynamics based on a gambling mechanism and its sensitivity analysis," Journal of Statistical Mechanics Theory \& Experiment, vol. 2017, no. 9, Article ID 093401, 2017.

[31] S. Wongkaew, M. Caponigro, and A. Borzì, "On the control through leadership of the Hegselmann-Krause opinion formation model," Mathematical Models and Methods in Applied Sciences, vol. 25, no. 3, pp. 565-585, 2015.

[32] J. Lorenz, "Heterogeneous bounds of confidence: meet, discuss and find consensus," Complexity, vol. 15, no. 4, pp. 43-52, 2010.

[33] J. Su, B. Liu, Q. Li, and H. Ma, "Coevolution of opinions and directed adaptive networks in a social group," Journal of Artificial Societies \& Social Simulation, vol. 17, no. 2, 2014.

[34] G. Fu, W. Zhang, and Z. Li, "Opinion dynamics of modified Hegselmann-Krause model in a group-based population with heterogeneous bounded confidence," Physica A: Statistical Mechanics and Its Applications, vol. 419, pp. 558-565, 2015.

[35] X. Chen, S. Zhao, and W. Li, "Opinion dynamics model based on cognitive styles: field-dependence and field-independence," Complexity, vol. 2019, Article ID 2864124, 12 pages, 2019.

[36] K. Tucci, J. C. González-Avella, and M. G. Cosenza, "Rise of an alternative majority against opinion leaders," Physica A: Statistical Mechanics and Its Applications, vol. 446, pp. 75-81, 2016.

[37] R. Albert and A.-L. Barabási, "Statistical mechanics of complex networks," Reviews of Modern Physics, vol. 74, no. 1, pp. 47-97, 2002.

[38] Y. Zhao, G. Kou, Y. Peng, and Y. Chen, "Understanding influence power of opinion leaders in e-commerce networks: an opinion dynamics theory perspective," Information Sciences, vol. 426, pp. 131-147, 2018.

[39] D. J. Watts and P. S. Dodds, "Influentials, networks, and public opinion formation," Journal of Consumer Research, vol. 34, no. 4, pp. 441-458, 2007.

[40] Y. Zhao, L. Zhang, M. Tang, and G. Kou, "Bounded confidence opinion dynamics with opinion leaders and environmental noises," Computers \& Operations Research, vol. 74, pp. 205-213, 2016.

[41] S. E. Parsegov, A. V. Proskurnikov, R. Tempo, and N. E. Friedkin, "Novel multidimensional models of opinion dynamics in social networks," IEEE Transactions on Automatic Control, vol. 62, no. 5, pp. 2270-2285, 2017.

[42] D. Zohar and O. Tenne-Gazit, "Transformational leadership and group interaction as climate antecedents: a social network analysis," Journal of Applied Psychology, vol. 93, no. 4, pp. 744-757, 2008.

[43] A. Mirtabatabaei and F. Bullo, "Opinion dynamics in heterogeneous networks: convergence conjectures and theorems," SIAM Journal on Control and Optimization, vol. 50, no. 5, pp. 2763-2785, 2012.

[44] Y. Moreno, M. Nekovee, and A. Pacheco, "Dynamics of rumor spreading in complex networks," Physical Review E: Statistical Nonlinear \& Soft Matter Physics, vol. 69, no. 2, pp. 279-307, 2004.

[45] H. Liang, Y. Yang, and X. Wang, "Opinion dynamics in networks with heterogeneous confidence and influence,"
Physica A: Statistical Mechanics and Its Applications, vol. 392, no. 9, pp. 2248-2256, 2013.

[46] Y. Wang, H. Li, J. Zuo, and Z. Wang, "Evolution of online public opinions on social impact induced by NIMBY facility," Environmental Impact Assessment Review, vol. 78, Article ID 106290, 2019.

[47] D. Middleton, Institute of Electrical and Electronics Engineers. An Introduction to Statistical Communication Theory, McGraw-Hill, New York, NY, USA, 1960. 